\title{
Etude théorique et expérimentale du fonctionnement en charge des canaux de fuite en l'absence de cheminée d'équilibre*
}

\author{
Theoretical and experimental investigations \\ of full-flowing tail-race tunnel behaviour \\ in the absence of a surge-tank
}

\author{
I. ESCANDE \\ CORRESPONDANT DE L'INSTTYT \\ INGÉNIEUR T.X.T. \\ ProfESSEUR A LA PACULTÉ DES SCLENCES Dr TOULOUSE \\ DMRECTEUR DE L'E.N.S.E.H.T.
}

PAR

$\mathrm{Fr}$

Etude theorique du fonctionnement d'un canal de fuite en charge lors d'une fermeture instantanée des turbines. - Comparaison des résultats (surpression, volume de la poche d'air, volume d'ean déversée obtenus soit par le calcul, soil par la méthode graphique Bercenon, dans les trois cas suivants: le canal de fuite ne comporte ancun dispositif d'aération; il comporte un clapet d'entrée d'air, système Caquor'; il comporte un puits d'áration. - Les résullats concordent el monlrent que, si des surpressions importantes risquent de prendre naissance, clapet ou puits sont à mème de les altinner dans une large mesure, le choix entre ces deux dispositifs étant affaire de circonstances. - Les pertes de charge ne diminuent que légèrement la surpression; cette derniere, par contre, diminue assez rapidement all fur et à mesare que la darée de fermeture des turbines augmente.

Ces diverses éfudes théoriques ont été soumises à un contrôle expérimental minutieux sux modèle réduit : la concordance est très satisfaisante dans les deux cas, canal non aéré et canal avec clapet; le cas du puits donne lieu $\dot{a}$ de petits écarts croissant avec les débits. Essais de visualisation du phénomène et observations sur un film à 1.700 images par seconde.

\section{J. NOUGARO}

INGÉNIEUR I.E.T.

DOCTEUR Ès SCIENCES

PROFESSEUR A L'E.X.S.E.H.T.

\begin{abstract}
Theoretical study of full-flouing tail-race tunnel behaviour when turbines are closed instantaneously. -- Comparison of the results (excess pressure, volumes of the air pocket and the water discharged) obtained in the following three cases either by calculation or by BerGenoN's graphical method: the tail-race, wilhout any aeration device; with a Caguot type, airintake valve; with an aeration shaft. The results conform and show that if there is a risk of considerable excess pressures appearing, the valve and shaft will, to a large extent, counteract this pressure; the choice between the two devices depends on the circumstances. Head losses will only slightly reduce the excess pressure; the latter will, to the contrary, diminish fairly rapidly with an increase of the period during which the lurbines are closed. These various theoretical studies have been carefully checked by experiment on a scale model. The conformity is very satisfactory in the two cases of a canal without aeration and with a valve; the case of the shaft gives rise to small differences which increase with the discharge. Tests for observation of the phenomenon and observations with a 1,700 images per second film were made.
\end{abstract}

\section{INTRODUCTION}

Les progrès de la Technique des Travaux Souterrains et ceux de la Mécanique des Sols ont eu une incidence très nette sur l'évolution de certaines tendances relatives à la conception des usi-

(*) Cette étude, dont une partie a déjà été publiée dans le Bulletin de la Société Française des Mécaniciens, a fait l'objet de deux communications à la Société Hydrotechnique de France, en juin 1952 et juin 1953. 
nes hydro-électriques et de leurs ouvrages.

Aujourd'hui, on adopte, chaque fois que la chose est possible, un canal d'amenée fonctionnant en charge, de préférence à un canal à ciel ouvert.

La salle des machines, elle-même, est très souvent souterraine, solution qui, en dehors des avantages évidents qu'elle présente en cas de guerre, correspond souvent à une solution économique.

Les Centrales souterraines comportent un canal de fuite, de longueur plus ou moins grande, qui, dans la plupart des cas, fonctionne en charge, en permanence, ou, tout au moins, lor's des crues provoquant une montée importante du plan d'eau dans la rivière au droit de la restitution.

En France, la Centrale de Brommat a été la première grande usine souterraine.

A titre d'exemple, nous citerons encore Paolo Afonso $(300.000 \mathrm{~kW}$ au. Brésil), et Jablanica (175.000 kW en Yougoslavie), parmi les Centrales pour lesquelles nous avons eu à effectuer des études, à l'étranger.

Dans la solution classique, on place, en tête du canal d'amenée en charge, une chemince d'équilibre, afin d'éviter la formation des coups de bélier d'onde.
Dans une étude antérieure, nous avons traité le cas où cette cheminée d'équilibre n'existe pas et montré comment une application judicieuse de la méthode graphique de SchnyderBERGERON permet de déterminer simultanément les surpressions dans la conduite forcée et dans le canal de fuite.

Nous avions laissé toutefois de côté, dans cette étude, le cas des grandes dépressions susceptibles de provoquer une cavitation dans le canal de fuite.

Nous avons tout récemment repris notre étude en envisageant tout spécialement l'hypothèse que nous avions ainsi laissée primitivement de côté et nous résumons les résultats que nous avons obtenus dans la présente étude.

Nous divisons notre exposé en trois parties: dans la première, qui fait l'objet du chapitre I, nous effectuons l'étude théorique du fonctionnement, en l'absence de cheminée d'équilibre, en négligeant les pertes de charge; dans la second partie (chapitre II), nous montrons comment il convient de tenir compte des pertes d'énergie; la dernière partie (chapitre III) est réservée à l'exposé des résultats expérimentaux qui confirme pleinement les prévisions théoriques.

\title{
ETUDE THEORIQUE \\ DU FONCTIONNEMENT EN CHARGE \\ DES CANAUX DE FUITE \\ EN L'ABSENCE DE CHEMINEE D'ÉQUILIBRE
}

\author{
(CAS ovi l'on NÉglige les pertes de charge)
}

\section{INTRODUCTION}

Comme il vient d'être dit, nous allons, dans le présent chapitre, examiner le cas d'un canal de fuite parcouru par l'eau à une vitesse assez grande pour que la dépression engendrée par le déclenchement provoque, soit une cavitation franche, en l'absence de dispositif d'aération, soit une entrée d'air importante dans le cas où celleci soit rendue possible.

Nous appliquerons les formules obtenues à un cas concret correspondant à une étude faite pour les Entreprises Métropolitaines et Coloniales :

- Longueur du canal de fuite. $\mathrm{L}=750 \mathrm{~m}$

- Section du canal de fuite.. $f=28,3 \mathrm{~m}^{2}$

- Débit dans le régime permanent initial............ $\mathrm{Q}_{0}=70 \mathrm{~m}^{3} / \mathrm{s}$ 
- Vitesse moyenne en régime permanent .......... W $W_{0}=2,48 \mathrm{~m} / \mathrm{s}$

- Pression statique, due au bief aval sur l'axe du canal. . . . $y_{0}=5 \mathrm{~m}$

- Vitesse de propagation des ondes ............. $a=1.250 \mathrm{~m} / \mathrm{s}$

-. Durée d'un aller retour d'onde $\ldots \ldots \ldots \ldots \ldots \ldots$ o $\theta=1,2 \mathrm{~s}$

Le schéma de la figure 1 précise les notations. Nous envisageons, en premier lieu, le cas où le canal ne possède aucun moyen d'aération.

Nous supposons ensuite que la galerie est munie d'un clapet permettant l'entrée de l'air extérieur dès que la pression tombe à une valeur très légèrement inférieure à la pression atmosphéri- que; ce clapet se referme aussitôt que la pression de l'air de la galcrie dépasse la pression atmosphérique.

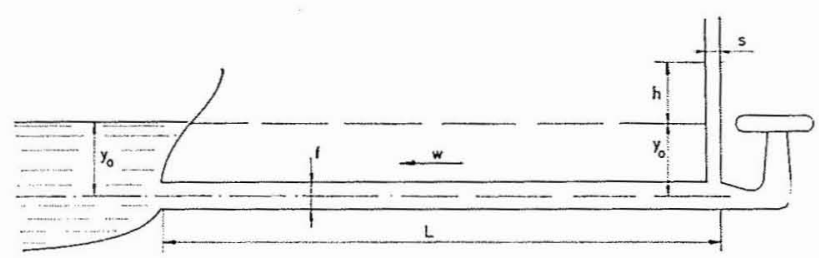

FIG. 1.

Enfin, nous supposons que le canal de fuite comporte un puits d'aération.

Dans les calculs, nous négligeons les pertes de charge, comme il a été indiqué.

\section{CANAL DE FUITE NE COMPORTANT AUCUNE POSSIBILITE D'AERATION FERMETURE INSTANTANEE}

Dès l'arrêt des turbines, il apparait, en tête du canal de fuite, une dépression égale à $-y_{a}, y_{a}$ désignant la pression atmosphérique : la pression tombe à la valeur du vide et une cavité prend naissance.

Sous l'effet de l'inertie, la masse d'eau en mouvement dans le canal de fuite poursuit, tout d'abord, son déplacement vers l'aval malgré la contrepression et l'accélération négative qui en résulte; la cavité augmente.

Au bout de quelques secondes, le mouvement vers l'aval s'arrête, puis l'eau se remet en vitesse vers l'amont, le volume de la cavité allant en diminuant.

Au moment où le volume atteint la valeur zéro, le mouvement de l'eau se trouve brusquement interrompu et une surpression importante prend naissance.

Le calcul peut être effectué soit à l'aide d'une méthode analytique, soit à l'aide d'une méthode. graphique.

\section{MÉthode ANALYTiQue}

Elle est basée sur la considération de l'oscillation en masse. Avec les notations précisées par la figure 1 , on peut écrire l'équation des forces vives du système :

$$
\frac{\mathrm{L}}{g} \frac{d \mathrm{~W}}{d t}+\left(y_{0}+y_{\theta}\right)=0
$$

On en déduit la vitesse de formation de la cavité :

$$
\mathrm{W}=\mathrm{W}_{0}-\frac{g}{\mathrm{~L}}\left(y_{0}+y_{a}\right) t
$$

Le volume de la cavité se détermine aisément :

$$
\Omega=\int_{0}^{t} f \cdot \mathrm{W} \cdot d t=f \mathrm{~W}_{0} t-\frac{g f}{2 \mathrm{~L}}\left(y_{0}+y_{a}\right) t^{2}
$$

Ce volume augmente jusqu'à l'instant $t_{1}$, tel que l'on ait $\mathrm{W}=0$, soit :

$$
t_{1}=\frac{\mathrm{L} \mathrm{W}_{0}}{g\left(y_{0}+y_{a}\right)}
$$

Le volume de la cavité est alors maximum et a pour valeur :

$\Omega_{1}=f \cdot \mathrm{W}_{0} \cdot t_{1}-\frac{g f}{2 \mathrm{~L}}\left(y_{0}+y_{a}\right) t_{1}{ }^{2}=\frac{\mathrm{L} f}{y_{0}+y_{a}} \cdot \frac{\mathrm{W}_{0}{ }^{2}}{2 g}$

On vérifie immédiatement que la cavité se referme entièrement en un temps $t_{2}$ égal à $t_{1}$ et que la vitesse du courant, à l'instant $t_{2}+t_{1}$, est égale à $W_{0}$, au signe près.

La surpression produite est donnée alors par l'expression :

$$
\xi_{\mathrm{MI}}=\frac{a \cdot \mathrm{W}_{0}}{g}
$$

L'application à l'exemple numérique étudié donne :

$$
\begin{aligned}
& t_{1}=12,62 \mathrm{~s}=10,50 \\
& \Omega_{1}=444 \mathrm{~m}^{3} \\
& \xi_{\mathrm{M}}=315 \mathrm{~m}
\end{aligned}
$$




\section{MÉTHODE GRAPHIQUE}

Nous avons appliqué au cas étudié la méthode graphique de Bergeron. La construction est reproduite sur la figure 2.

L'unité de temps utilisée est la durée o d'un aller et retour d'onde dans le canal.

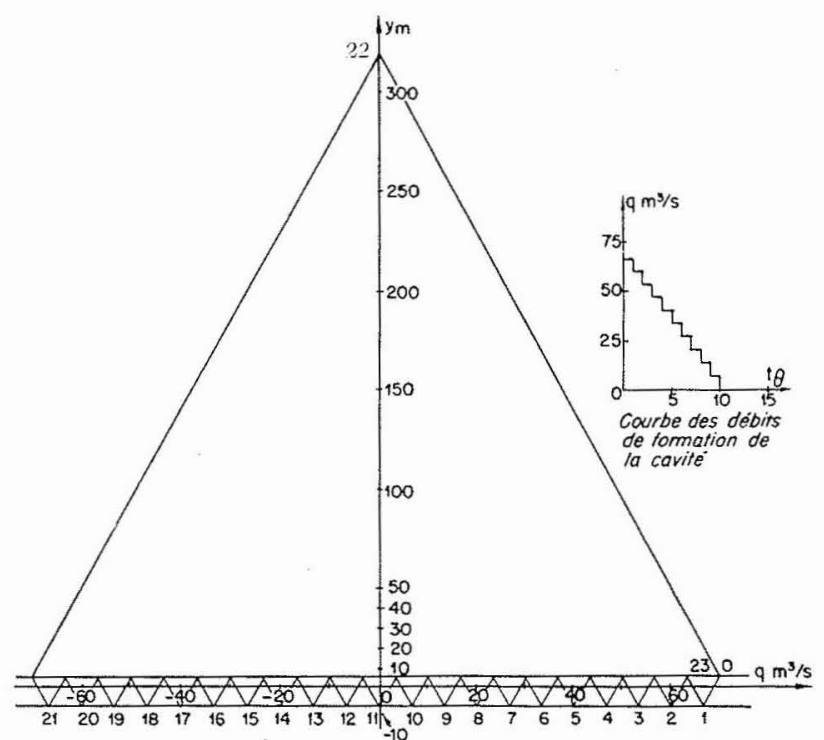

Fig. 2. - Canal de fuite ne comportant pas de clapet ou de puits d'aćration. Etude d'une fermeture instantanée des turbines.

A l'instant initial, le point de fonctionnement se trouve en 0 (pression statique $y_{0}=5 \mathrm{~m}$, débit $\left.\mathrm{Q}_{0}=70 \mathrm{~m}^{3} / \mathrm{s}\right)$.

A partir de la fermeture instantanée, un lieu de tous les points de fonctionnement est fourni par la droite $y=-y_{\imath}$. Le premier point se trouve en outre sur la droite de pente $a / g f$, en 1.
Selon la construction classique, le point de fonctionnement à l'instant 2 se trouve sur le systèmes de droites, $\varphi$ et $\Phi$, réfléchi sur l'axe des pressions statiques, à partir du point 1 . Le deuxième lieu étant toujours la droite de cavitation, leur intersection fournit la position du point 2 .

On passe de la même façon de 2 à 3 , de 3 à 4 et ainsi de suite. On peut suivre ainsi la formation de la cavité qui s'effectue avec des débits connus, aux instants 1, $2 \ldots$ L'intégration de la courbe de ces débits (fig. 2) fournit le volume maximum $\Omega_{1}$ de la cavité.

La construction se poursuit avec des valeurs négatives du débit et la cavité se résorbe.

A l'instant $t_{1}+t_{2}$ où elle est entièrement résorbée, on obtient une surpression brusque, le lieu des points de fonctionnement devenant, sur le graphique, la droite $q=0$.

Avec l'exemple numérique envisagé, la construction est symétrique par rapport à l'axe des surpressions : le point de surpression maximum est en 22.

Il revient ensuite en 0 et la cavité se reforme.

Le phénomène est périodique, dans l'hypothèse où nous nous sommes placés, de pertes de charge nulles. La période est égale à 230 , soit $27,6 \mathrm{~s}$.

La construction donne :

$$
\begin{aligned}
t_{1} & =10,50 \\
\Omega_{1} & =442 \mathrm{~m}^{3} \\
\xi_{\mathrm{M}} & =315 \mathrm{~m}
\end{aligned}
$$

valeurs pratiquement égales à celles qui ont été obtenues avec le calcul d'oscillation en masse.

\section{CANAL DE FUITE COMPORTANT UN CLAPET FERMETURE INSTANTANEE}

Sur la demande de M. le Président Caguor, nous avons examiné le cas où le canal de fuite est muni, à la sortie des turbines, d'un clapet dont l'ouverture se produit dès que la pression dans le canal tombe à une valeur inférieure à la pression atmosphérique $y_{a}$. Ce clapet se referme à partir du moment où la pression dans le canal devient supérieurre à cette même valeur. Pour simplifier le calcul, nous supposons que ce clapet, idéalement sensible, s'ouvre ou se ferme pour des valeurs de la pression infiniment voisines, de part et d'autre de la pression atmosphérique, leur différence avec cette dernière étant négligeable, en première approximation.
Le mécanisme des phénomènes qui interviennent dans le canal de fuite demeure analogue au précédent, dans ses grandes lignes: la masse d'eau en mouvement vers l'aval continue son déplacement; la pression tombe à une valeur égale à $y_{t}$, le clapet s'ouvre, l'air pénètre et occupe un volume qui augmente tout d'abord. Au bout de quelques instants, le mouvement vers l'aval s'arrête, l'eau se remet en mouvement vers l'amont et comprime l'air emprisonné par la fermeture du clapet.

La surpression maximum est atteinte au moment où le volume est réduit à sa valeur minimum. 
Le calcul peut ètre effectué, comme précédemment, soit à l'aide d'une méthode analytique, soit a livide d'une méthode graphique.

\section{MÉTHODE ANALYTIOUE}

L'équation des forces vives s'écrit maintenant:

$$
\frac{\mathrm{L}}{g} \cdot \frac{d \mathrm{~W}}{d t}+y_{0}=0
$$

On en déduit la vitesse de formation de la bulle d'air :

$$
\mathrm{W}=\mathrm{W}_{0}-\frac{g y_{0}}{\mathrm{~L}} t
$$

Le volume $\varrho$ de la cavité est ainsi :

$$
\Omega=\int_{0}^{t} f \cdot \mathrm{W} \cdot d t=f \cdot \mathrm{W}_{0} t-\frac{g f}{2 \mathrm{~L}} y_{0} t^{2}
$$

Ce volume est maximum jusqu’à l'instant $t_{1}=:=\left(\mathrm{L} \mathrm{W}_{0}\right) /\left(g y_{0}\right)$ pour lequel la vitesse s'annule.

Le volume de la cavité est alors :

$$
\Omega_{1}=\frac{\mathrm{L} f}{y_{0}} \cdot \frac{\mathrm{W}_{0}{ }^{2}}{2 g}
$$

A partir de cet instant $t_{1}$, la cavité se referme.

Ecrivons l'équation des forces vives, la loi de Mariotrte et l'expression de la dérivée du volume $\Omega$ de la poche d'air, de pression $y$ :

$$
\begin{gathered}
\frac{\mathrm{L}}{g} \frac{d \mathrm{~W}}{d t}=-y_{0}+y \\
\left(y+y_{a}\right) \Omega=y_{a} \Omega_{1} \\
\frac{d \varrho}{d l}=f \mathrm{~W}
\end{gathered}
$$

On en tire :

$\frac{\mathrm{L}}{g} \cdot \frac{d \mathrm{~W}}{d \Omega} \cdot \frac{d \Omega}{d t}=\frac{\mathrm{L} /}{g} \mathrm{~W} \frac{d \mathrm{~W}}{d \Omega}=-y_{0}+\eta$

et en remplaçant $y$ par sa valeur :

$$
-y_{0}-y_{a}+y_{a} \frac{\Omega_{1}}{\Omega}=\frac{\mathrm{L} f}{g} \mathrm{~W} \frac{d \mathrm{~W}}{d \Omega}
$$

On en déduit la valeur de la vitesse $\mathrm{W}$ :

$$
\mathrm{W}^{2}=\frac{2 g}{\mathrm{~L} /}\left[\left(y_{0}+y_{1}\right)\left(\Omega_{1}-\Omega\right)-y_{a} \Omega_{1} \log \frac{\Omega_{1}}{\Omega}\right]
$$

La vitesse s'annule à l'instant $t_{1}+t_{2}$ pour un volume $\Omega=\Omega_{2}$ tel que :

$$
\left(y_{0}+y_{a}\right)\left(\Omega_{1}-\Omega_{2}\right)=y_{a} \Omega_{1} \log \frac{\Omega_{1}}{\Omega_{2}}
$$

équation qui donne $\Omega_{.2}$; on en déduit la pression maximum $y_{2}$ :

$$
y_{2}=y_{a}\left[\frac{\Omega_{1}}{\Omega_{2}}-1\right]
$$

L'application à l'exemple numérique étudié donne

$$
\begin{aligned}
& \Omega_{1}=1.332 \mathrm{~m}^{3} \\
& \Omega_{2}=551 \mathrm{~m}: \\
& y_{2}=14,18 \mathrm{~m}
\end{aligned}
$$

\section{MÉTHOde GRAPHIQUe}

La construction graphique est identique, au début, à celle que nous avons indiquée au chapitre précédent. La seule différence réside dans le fait que le lieu des points de fonctionnement n'est plus la droite de cavitation $y=-y_{a}$, mais la droite $y=-y_{0}$.

Lorsque le débit change de signe, la poche d'air commence à se refermer. Dès cet instant, le point $n$ se trouve sur le système des droites $\varphi, \Phi$ réfléchies sur l'axe des débits à partir du point $n-1$, et l'on a, de plus :

$$
\left(y_{n}+y_{n}\right) \Omega_{n}=y_{a} \cdot \Omega_{1}
$$

ce qui détermine $y_{n}$ et donne la position du point $n$, en opérant par approximations successives.

Dans le cas que nous étudions, la construction graphique est reproduite sur la figure 3.

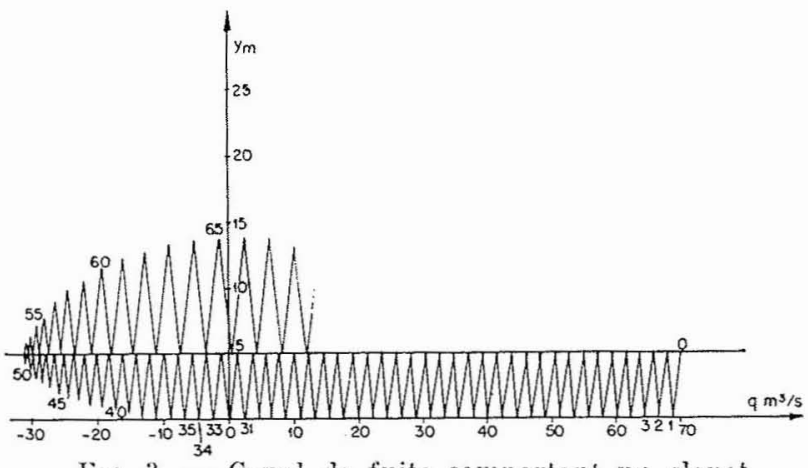

Fiti. 3. - Canal de fuite comportant un clapet. Etude d'une fermeture instantanée des turbines.

On obtient les résultats suivants :

$$
\begin{aligned}
& \Omega_{1}=1.340 \mathrm{~m}^{3} \\
& \Omega_{2}=551 \mathrm{~m}^{3} \\
& y_{2}=13,90 \mathrm{~m}
\end{aligned}
$$

valeurs très voisines des résultats obtenus pour le calcul d'oscillations en masse. 


\section{CANAL DE FUITE COMPORTANT UN PUITS D'AERATION FERMETURE INSTANTANEE}

Le canal de fuite est muni, à lamont, d'un puits d'aération permettant une entrée d'air en cas d'aspiration. Le fonctionnement du système est analogue aux précédents. Dès l'arrêt des turbines, l'eau en mouvement vers l'aval continue son déplacement; dès que la pression tombe à une valeur égale à $y_{a}$, pression atmosphérique, l'entrée de l'air se produit par le puits. Une poche se forme, dont le volume augmente. Au bout de quelques secondes, le mouvement vers l'aval s'arrête, l'eau se remet en mouvement ver's l'amont et le volume de la poche d'air diminue. Il devient nul à un certain instant et la surpression qui se produit provoque un déversement par le puits d'aćration, si la hauteur de ce dernier n'est pas suffisante.

On peut toujours effectuer le calcul soit à l'aide de la méthode analytique, soit à l'aide de. la méthode graphique.

\section{MÉthode anaLytioue}

L'équation des forces vives s'écrit:

$$
\frac{\mathrm{L}}{g} \frac{d \mathrm{~W}}{d t}+y_{0}=0
$$

Le calcul est donc rigoureusement identique, au début, à celui que nous avons indiqué pour l'étude du canal de fuite muni d'un clapet.

Le volume de la cavité :

$$
\Omega=f \mathrm{~W}_{0} t-\frac{g f}{2 \mathrm{~L}} y_{0} t^{2}
$$

est maximum à l'instant $t_{1}$ :

$$
t_{1}=\frac{\mathrm{L} \mathrm{W}}{g y_{0}}
$$

et a alors pour valeur :

$$
\Omega_{1}=\frac{\mathrm{L} f}{y_{0}} \cdot \frac{\mathrm{W}_{0}{ }^{2}}{2 g}
$$

On vérifie immédiatement que la poche se referme en un temps $t_{2}$ égal à $t_{1}$ et que la vi- tesse du courant à l'instant $t_{1}+t_{2}$ est égale à $\mathrm{W}_{0}$, au signe près.

Le débit qui arrive alors est égal à $Q_{0}$ et son passage à travers la cheminée, assimilable à un ajutage, de section utile $m \mathrm{~S}$, nécessite une pression :

$$
y_{2}=y_{0}+h+\frac{\mathrm{Q}_{0}^{2}}{2 g m^{2} \mathrm{~S}^{2}}
$$

$h$ désignant la hauteur de la cheminée déversante au-dessus du niveau statique aval $\left(^{*}\right)$.

On peut déterminer le volume déversé par le puits. A partir de l'instant $t_{1}+t_{2}$, où s'établit la pression $y_{2}$, l'équation des forces vives s'écrit :

$$
\begin{aligned}
\frac{\mathrm{L}}{g} \frac{d \mathrm{~W}}{d t} & =-y_{0}+y_{2}=h+\frac{\mathrm{Q}_{0}^{2}}{2 g \mathrm{~m}^{2} \mathrm{~S}^{2}} \\
& =h+\frac{f^{2}}{2 g m^{2} \mathrm{~S}^{2}} \mathrm{~W}^{2}
\end{aligned}
$$

que l'on peut écrire :

$$
d t=\frac{\mathrm{L}}{g} \frac{d \mathrm{~W}}{h+\frac{f^{2} \mathrm{~W}^{2}}{2 g m^{2} \mathrm{~S}^{2}}}
$$

L'intégration de cette relation conduit à la valeur suivante de la vitesse :

$$
\mathrm{W}=\frac{m \mathrm{~S}}{f} \sqrt{2 g h} \mathrm{tg}\left[\left(t-t^{\prime}\right) \frac{f}{2 \mathrm{LmS}} \sqrt{2 g h}\right]
$$

Comme à l'instant $t_{0}=t_{1}+t_{2}, \mathrm{~W}=-\mathrm{W}_{0}$, on en déduit, en posant : $\mathrm{K}=\frac{m \mathrm{~S}}{f} \sqrt{2 g h}$ :

$$
\mathrm{W}=\mathrm{K} \operatorname{tg}\left[\frac{g h}{\mathrm{KL}}\left(t-t_{0}\right)-\operatorname{Arctg} \frac{\mathrm{W}_{0}}{\mathrm{~K}}\right]
$$

Le débit déversé est, à l'instant $t$ :

$q=f \mathrm{~W}=f \mathrm{~K} \operatorname{tg}\left[\frac{g h}{\mathrm{KL}}\left(t-t_{0}\right)-\operatorname{Arctg} \frac{\mathrm{W}_{0}}{\mathrm{~K}}\right]$

${ }^{\star}$ ) Le coefficient $m$ peut éventuellement tenir compte de la présence d'un étranglement placé à la base de la cheminée. 
Le volume déversé est donc :

$$
\Omega^{\prime}=\int_{t_{0}}^{t_{0}+t_{3}} q \cdot d t
$$

en appelant $t_{1}+t_{2}+t_{3}$ l'instant où le déversement s'arrête, soit :

$\alpha^{\prime}=\frac{\mathrm{K}^{2} f \mathrm{~L}}{g h} \cdot \log \cos \left[\frac{g h}{\mathrm{KL}}\left(t-t_{0}\right)-\operatorname{Arctg} \frac{\mathrm{W}_{0}}{\mathrm{~K}}\right]_{t_{0}}^{t_{0}+t_{3}}$ $t_{\text {g }}$ étant égal à $\frac{\mathrm{KL}}{g h} \operatorname{Arctg} \frac{\mathrm{W}_{0}}{\mathrm{~K}}$.

On obtient ainsi successivement :

$$
\begin{aligned}
& \Omega^{\prime}=\frac{\mathrm{K}^{2} f \mathrm{~L}}{g h} \log \left(\cos \operatorname{Arctg} \frac{\mathrm{W}_{0}}{\mathrm{~K}}\right) \\
& \Omega^{\prime}=\frac{\mathrm{K}^{2} f \mathrm{~L}}{g h} \log \left|\frac{\sqrt{\mathrm{K}^{2}+\mathrm{W}_{0}^{2}}}{\mathrm{~K}}\right|
\end{aligned}
$$

ou, en remplaçant $\mathrm{K}$ par sa valeur :

$$
\Omega^{\prime}=\frac{\mathrm{L} m^{2} \mathrm{~S}^{2}}{f} \log \left[1+\frac{f^{2}}{m^{2} \mathrm{~S}^{2}} \frac{\mathrm{W}_{0}{ }^{2}}{2 g h}\right]
$$

L'application de la relation donnant la valeur de $q$ en fonction de $t$ permet de tracer une courbe dont l'intégration graphique fournit également la valeur de $\Omega$.

\section{MÉthode GRAPHIQUE}

La méthode utilisée est identique à celle que nous avons indiquée pour l'étude du canal de fuite muni d'un clapet.

Le point de fonctionnement à l'instant $n$ se trouve à l'intersection de la droite de pression atmosphérique $y=-y_{0}$ et du système de droites $\oplus$ Ф réfléchi sur l'axe des pressions statiques, à partir du point $(n-1)$.

Elle se poursuit pour les débits négatifs, jusqu'au moment où la poche d'air se résorbe.

A cet instant, on obtient une surpression brusque, le lieu des points de fonctionnement devenant la parabole :

$$
y=y_{0}+h+\frac{q^{2}}{2 g m^{2} \mathrm{~S}^{2}}
$$

La figure 4 reproduit la construction graphique correspondant à l'un des cas étudiés (section utile $m \mathrm{~S}=6 \mathrm{~m}^{2}$, hauteur du puits $h=5 \mathrm{~m}$ ). Cette construction permet de suivre le volume d'eau déversé, à partir de l'instant où naît la surpression.

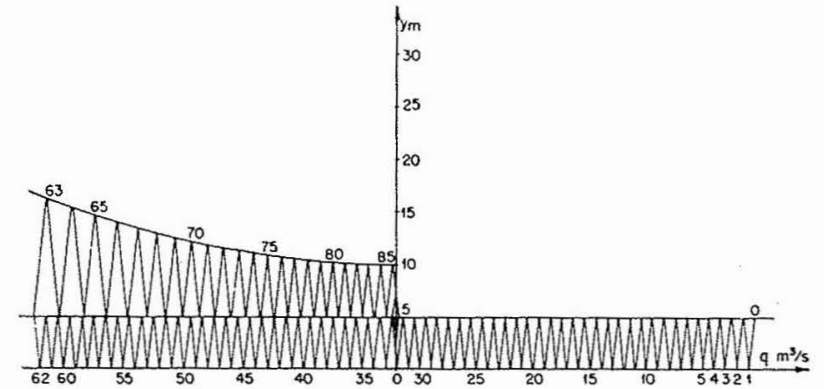

Fig. 4. - Canal de fuite comportant un puits d'aération : $h=5 \mathrm{~m} \quad m \mathrm{~S}=6 \mathrm{~m}^{2}$

Etude d'une fermeture instantanée des turbines.

\begin{tabular}{|c|c|c|c|c|c|c|c|c|}
\hline \multirow{2}{*}{$\begin{array}{c}\begin{array}{c}\text { Section } \\
\text { utile }\end{array} \\
m \mathrm{~S} \\
\mathrm{~m}^{2}\end{array}$} & \multicolumn{2}{|c|}{$\underbrace{h=0}_{\begin{array}{c}\text { Surpression } \\
\mathrm{m}\end{array}}$} & \multicolumn{2}{|c|}{$\underbrace{h=1 \mathrm{~m}}_{\begin{array}{c}\text { Surpression } \\
\mathrm{m}\end{array}}$} & \multicolumn{2}{|c|}{$\underbrace{h=3 \mathrm{~m}}_{\substack{\text { Surpression } \\
\mathrm{m}}}$} & \multicolumn{2}{|c|}{$\underbrace{h=5 \mathrm{~m}}_{\substack{\text { Surpression } \\
\mathrm{m}}}$} \\
\hline & 总 & 遌 & 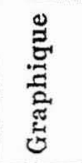 & 离 & 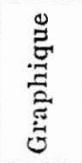 & 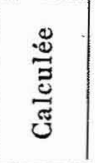 & 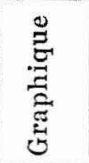 & 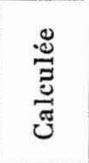 \\
\hline 2 & 45 & 62,4 & 46,4 & 63,4 & 48 & 65,4 & 49 & 67,6 \\
\hline 4 & 14,3 & 15,6 & 15,2 & 16,6 & 17,2 & 18,6 & 18,8 & 20,6 \\
\hline 6 & 6,6 & 6,95 & 7,6 & 7,95 & 9,5 & 9,95 & 11,4 & 11,95 \\
\hline 8 & 3,85 & 3,91 & 4,8 & 4,91 & 6,8 & 6,91 & 8,7 & 8,91 \\
\hline
\end{tabular}

Le tableau suivant résume, en ce qui concerne la surpression maximum, les résultats obtenus par le calcul analytique et la construction graphique.

Ces valeurs sont très voisines les unes des autres, le cas du puits de $2 \mathrm{~m}^{2}$ de section utile présentant néanmoins des différences assez sensibles entre les valeurs déduites des graphiques et calculées. Cette différence peut s'expliquer par le fait que nous admettons que le débit du puits est égal à $Q_{0}$ au moment où se produit la surpression maximum. Or, comme le montre la construction graphique, le débit à cet instant est différent de $Q_{0}$, cette différence étant assez élevée pour le cas $m \mathrm{~S}=2 \mathrm{~m}^{2}$, alors qu'elle diminue rapidement quand 'la section utile du puits augmente, comme le montre le tableau suivant mettant en évidence la valeur maximum du débit du puits : 


\begin{tabular}{|c|c|c|c|c|c|c|c|c|}
\hline \multirow[b]{2}{*}{$\begin{array}{c}\text { Section } \\
\text { utile } \\
m \mathrm{~S}\end{array}$} & \multicolumn{2}{|c|}{$h=0$} & \multicolumn{2}{|c|}{$h=1 \mathrm{~m}$} & \multicolumn{2}{|c|}{$h=3 \mathrm{~m}$} & \multicolumn{2}{|c|}{$h=5 \mathrm{~m}$} \\
\hline & 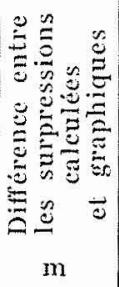 & 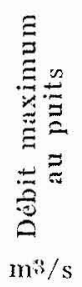 & 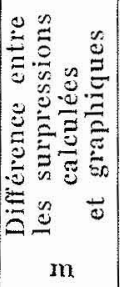 & 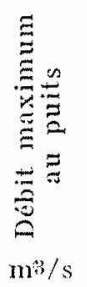 & 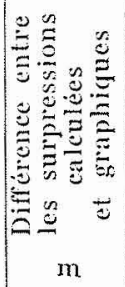 & 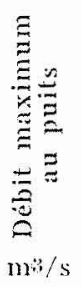 & 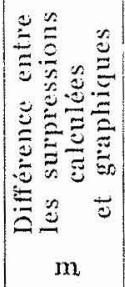 & 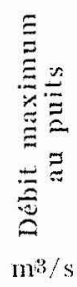 \\
\hline 2 & 17,4 & 59,8 & 17 & 59,4 & 17,4 & 59,2 & 18,4 & 59,0 \\
\hline 4 & 1,3 & 66,8 & 1,4 & 66,6 & 1,4 & 66,4 & 1,8 & 65,8 \\
\hline 6 & $0,3 \tilde{5}$ & 68,5 & 0,35 & 68,0 & 0,45 & $6 \pi, 8$ & 0,55 & 67,4 \\
\hline 8 & 0,06 & 68,1 & 0,11 & $(68,9$ & 0,11 & 68,4 & 0,21 & $(68,0)$ \\
\hline
\end{tabular}

On peut faire également la comparaison entre les volumes déversés, déduits des constructions et du calcul analytique; le tableau suivant résume ces résultats.

\begin{tabular}{|c|c|c|c|c|c|c|}
\hline \multirow{3}{*}{$\begin{array}{c}\text { Section } \\
\text { utile } \\
m \mathrm{~S} \\
\mathrm{~m}^{2}\end{array}$} & \multirow{2}{*}{\multicolumn{2}{|c|}{$h=1 \mathrm{~m}$}} & \multirow{2}{*}{\multicolumn{2}{|c|}{$\underbrace{h=3 \mathrm{~m}}_{\text {Volume } \mathrm{m} 3}$}} & \multirow{2}{*}{\multicolumn{2}{|c|}{ Volume $\mathrm{m}^{3}$}} \\
\hline & & & & & & \\
\hline & 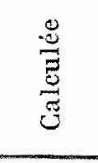 & 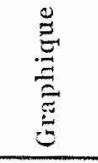 & 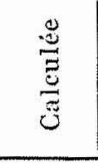 & 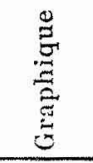 & $\frac{.0}{3}$ & 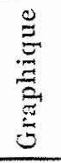 \\
\hline 2 & 440 & 420 & 326 & 322 & 276 & 280 \\
\hline 4 & 1.190 & 1.120 & 778 & 792 & 602 & 592 \\
\hline 6 & 1.970 & 1.970 & 1.145 & 1.112 & 832 & 832 \\
\hline 8 & 2.700 & 2.500 & 1.450 & 1.390 & 989 & 972 \\
\hline
\end{tabular}

Le cas $h=0$ 'n'est pas à envisager, puisque le calcul analytique, aussi bien que la construction graphique, montrent que la durée de déversement et le volume déversé tendent vers l'infini.

Pour les autres cas, les chiffres fournis par les deux méthodes sont en bonne concordance.
En particulier, ceux qui correspondent au cas d'une section utile $m \mathrm{~S}=2 \mathrm{~m}^{2}$ ne reflètent plus des différences comparables à celles que nous avions trouvées pour les surpressions. Ceci s'ex-

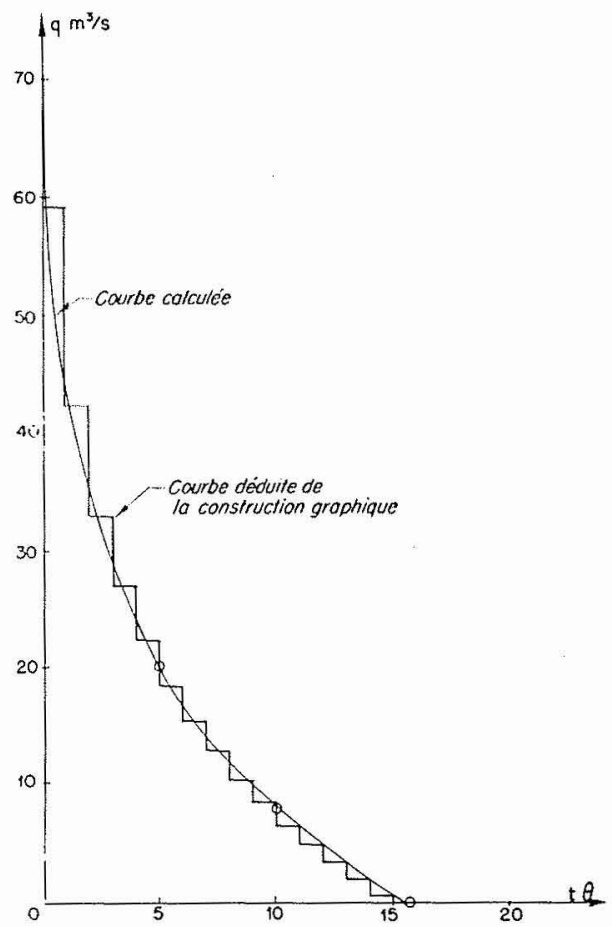

Fig. 5. - Courbes du débit déversé pour un puits : $h=3 \mathrm{~m} \quad m \mathrm{~s}=2 \mathrm{~m}^{2}$

plique par l'examen de la figure $n^{\circ} 5$ qui fournit dans l'un des cas étudiés $\left(h=3 \mathrm{~m}, \mathrm{mS}=2 \mathrm{~m}^{2}\right)$ 
les courbes du débit déduites de la construction et du calcul. Le calcul analytique suppose que te débit au départ est le débit maximum $70 \mathrm{ml}^{3} / \mathrm{s}$; la construction le donne plus faible, mais la surface correspondant à la différence est très faible ct n'influe nullement sur le résultat final.

Cette figure permet de se rendre compte de la liès bonne concordance entre les deux courbes.

Les résultats obtenus permettent de se rendre compte de l'influence d'un étranglement placé à la base de la cheminée el réduisant la valeur do $m$ : toutes choses égales d'ailleurs, la présence de cet étranglement augmente la surpression mais réduit le volume déversé.

\section{INFLUENCE DE LA DUREE DE FERMETURE DES TURBINES}

Dans le cas d'une fermeture d'une certaine durée, le débit varie suivant une loi qui dépend à la fois de la vitesse de fermeture du distributeur et du régime de surpressions qui s'établit dans la conduite forcée et dans le canal de fuite. Le calcul rigoureux pourrait être fait à partir de la méthode rappelée au début de ce chapitre. Pour simplifier, notre but étant seulement de mettre en évidence l'importance du facteur duréc de fermeture, nous supposerons, dans ce chapitre, que le débit des turbines passe linéairement de $Q_{0}$ à 0 en $\tau$ secondes.

Pratiquement, tant que la durée $\tau$ reste relativement faible, on peut admettre pour le calcul analytique, en première approximation, que le volume de la cavité, ou de la bulle d'air, dans le cas d'aération, differe seulement de celui que l'on obtient en fermeture instantanée, du volume total d'eau provenant des turbines pendant la durée $\tau$, soit $\approx Q_{0} / 2$.

Pour ce calcul analytique de la surpression, on a alors, $t_{1}$ et $\Omega_{1}$ restant ceux qui ont été déterminés en fermeture instantanée, le mème système d'ëquation, sauf à remplacer $\Omega_{1}$ par $\Omega_{1}-\tau Q_{0} / 2$.

Les constructions graphiques peuvent, par contre, être modifiées, pour suivre exactement la marche du phénomine.

Le lieu des points de fonctionnement est la droite $q=q_{n}$ représentant à l'instant $n$ la valeur du débit qui sort des turbines. Le svstème des droites $\Phi \Phi$ rencontre cette droite au point de fonctionnement $n$, à moins qu'elle coupe, avant cette intersection, la droite de cavitation ou de pression atmosphérique. La pression ne pouvant descendre au-dessous de cette valeur, on obtient ainsi le point de fonctionnement $n$. Par ailleurs, la droite $q=q_{n}$ a comme abscisse le débit $q_{n}$ des turbines à l'instant $n$.

Nous allons examiner les trois cas que nous avons envisages pour l'étude des f'ermetures instantanées.

\section{1. - Canal de fuite ne comportant AUCUNE POSSIBILITÉ D'AÉRATION}

Le calcul analytique de la surpression a été fait, en prenant les mèmes équations que dans le cas de la fermeture instantance, sauf à remplacer $\Omega_{1}$ par $\Omega_{1}-\tau Q_{0} / 2$.

En admettant une durée de fermeture : inférienre a la valeur $t_{1}$, temps au bout duquel la cavité a son volume maximum, on obtient une surpression donnée par l'expression :

$$
\zeta_{\mathrm{MI}}=\frac{a \mathrm{~W}_{.}}{g}
$$

Seule, la valeur de $W_{2}$ est différente de celle obtenue pour une manueuvre instantanée:

$$
W_{2}=\sqrt{W_{0}^{2}-\frac{Q_{0}}{2} \tau 2 g \frac{y_{0}+y_{a}}{f L_{2}}}
$$

Nous avons étudié graphiquement la formation de la cavité dans le canal de fuite, pour des durées de fermeture du distributeur des turbines respectivement igales à $60,10,50$ et 150 .

Seul, le cas de durée $\tau=60$ a été étudié par le calcul analytique. L'application des relations précédentes donne une surpression :

$$
\xi_{\mathrm{x}}=212 \mathrm{~m}
$$

Les constructions graphiques sont reproduites sur les figures 6, 7, 8. Ces mèmes figures donnent les courbes de débit en fonction du temps et montrent le processus de formation de la cavilé.

Le tableau suivant résume les résultats que

\begin{tabular}{|c|c|c|}
\hline \multirow{2}{*}{$\begin{array}{c}\text { Temps } \\
\text { de fermeture } \\
\tau\end{array}$} & $\begin{array}{l}\text { Surpression } \\
\text { maximum }\end{array}$ & \multirow{2}{*}{$\begin{array}{l}\text { Volume maximum } \\
\text { de la cavité } \\
\Omega_{1} \mathrm{~m}^{3}\end{array}$} \\
\hline & $\xi_{\mathrm{M}} \quad \mathrm{m}$ & \\
\hline $7,2 \mathrm{~s}=60$ & 222,5 & 198,73 \\
\hline $12,6 \mathrm{~s}=10,50$ & 135 & 17,1 \\
\hline $18 \mathrm{~s}=150$ & 10 & 0,38 \\
\hline
\end{tabular}
nous avons déduits du calcul analylique des constructions. 


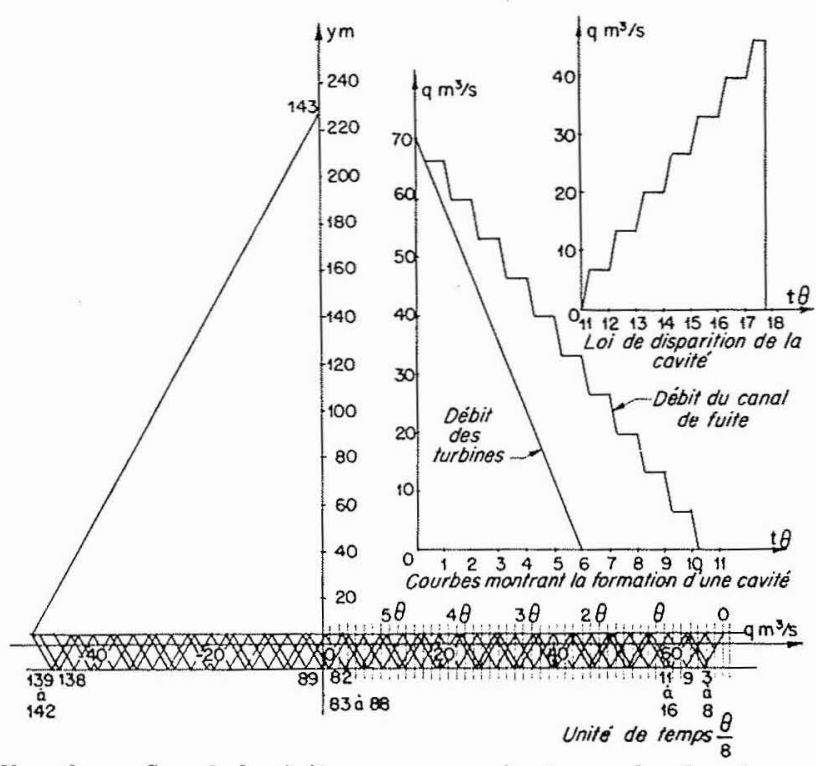

Fig. 6. - Canal de fuite ne comportant pas de clapet ou de puits d'aćration. - Etude d'une fermeture linéaire des turbines de durce $\tau=6 \vartheta$.

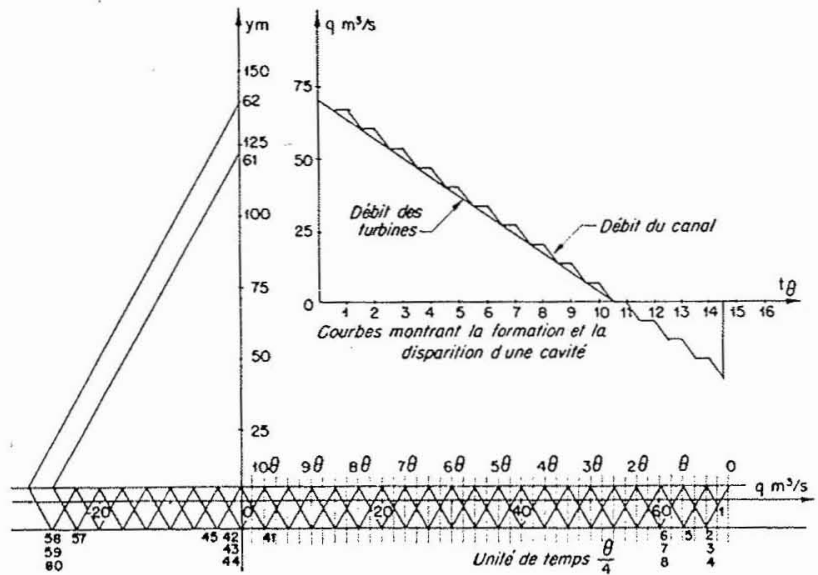

FiG. 7. - Canal de fuite ne comportant pas de clapet ou de puits d'aćration. - Etude d'une fermeture linéaire des turbines de durée $\tau=10,5 \vartheta$.

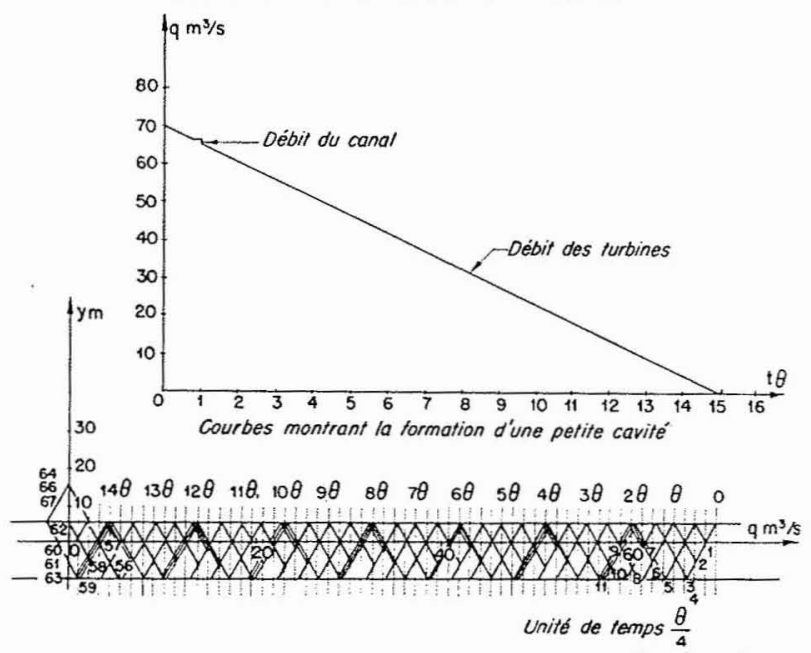

Fig. 8. - Canal de fuite ne comportant pas de clapet ou de puits d'aération. - Etude d'une fermeture linéaire des turbines de durée $\tau=15 \vartheta$.
Les chiffres obtenus pour la durée de fermeture $\tau=6 \theta$ montrent, pour une cavitation franche, la bonne concordance entre les valeurs obtenues par la méthode analytique et la méthode graphique.

On voit que le volume de la cavité (fig. 8) diminue très vite quand la durée de fermeture augmente. Pour le dernier cas étudié, le volume est pratiquement négligeable : nous sommes à la limite de la cavitation.

De même, la valeur de la surpression maximum diminue très rapidement quand la durée de fermeture augmente (fig. 9).

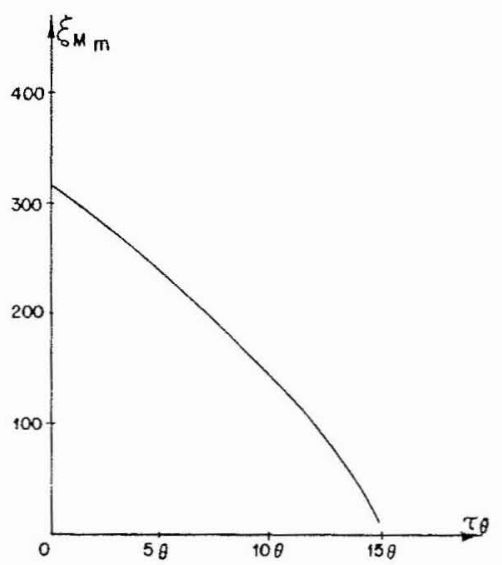

FiG. 9. - Canal de fuite ne comportant pas de clapet ou de puits d'aération.

Courbe de la surpression maximum en fonction de la durcée de fermeture des turbines.

\section{2. - Canal de fuite comportant un clapet.}

Le calcul analytique de la surpression a été fait en prenant, $t_{1}$ et $\Omega_{1}$ restant les mêmes que dans le cas de la fermeture instantanée, le même
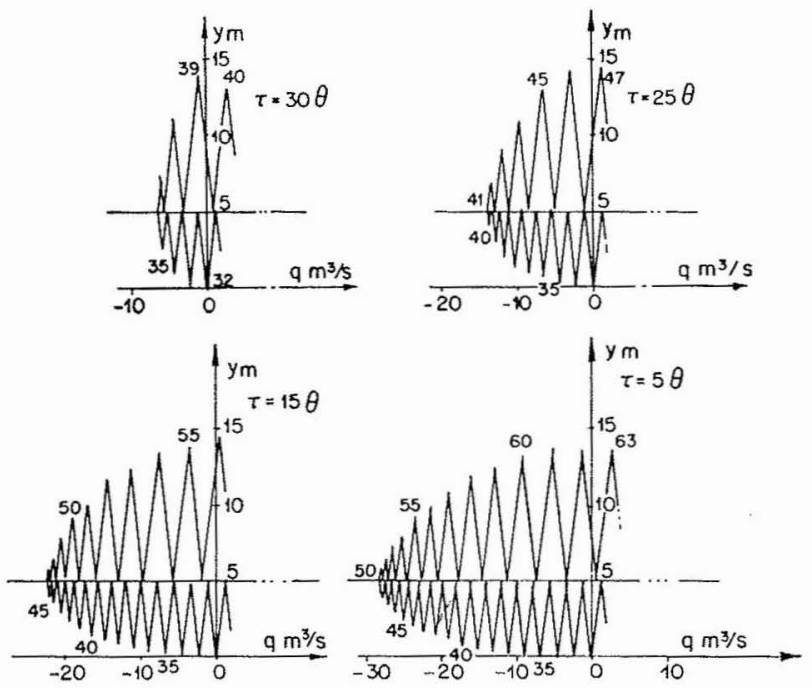

Fiti. 10. - Canal de fuite comportant un clapet.

Etude de fermetures linéaires des turbines de durée $\tau$. 
système d'équations, sauf à remplacer $\Omega_{1}$ par $\mathrm{O}_{1},-\mathrm{Q}_{0} / 2$.

Le calcul et les constructions graphiques ont 6lé effectués pour des valeurs de $\tau$ égales à 50 , 150,250 et 300 .

La figure $n^{\circ} 10$ reproduit la partie de la conslruction relative à ces quatre cas, à partir du moment où le clapet commence à se refermer. La première partie est en effet identique à celle qui correspond à l'étude de la fermeture instanlanée du distributeur. Ces calculs et constructions ont donné les résultats suivants :

\begin{tabular}{|c|c|c|c|c|c|}
\hline $\begin{array}{c}\text { Durée } \\
\text { de fermeture } \\
\tau\end{array}$ & 0 & 50 & 150 & 250 & 300 \\
\hline$\Omega_{1}-35 \tau \mathrm{m}^{*}$ & 1.332 & 1.123 & 702 & 282 & 72 \\
\hline$\Omega_{1}$ calcule $\mathrm{m}^{3}$ & 551 & 463 & 292 & 116 & 29,9 \\
\hline$\xi_{\mathrm{NI}}$ calculé m & 9,18 & 9,30 & 9,05 & 9,15 & 9,10 \\
\hline s.graphique m & 8,90 & 9,20 & 9,20 & 9,20 & 8,80 \\
\hline
\end{tabular}

Ces chiffres montrent que les valeurs obtenues par la méthode analytique et par la méthode graphique sont très voisines. La surpression est pratiquement constante pour des durćes de fermeture allant jusqu'à $30 \mathrm{~s}(25 \theta)$ : elle ne diminue de facon notable que pour des temps de fermeture supérieur's à 300 .

Le volume de la bulle d'air diminue, au contraire, fortement lorsque la durée de fermeture augmente.

\section{3. - Canal De Fuite comportant un puits D'AÉRATION.}

Comme précédemment, nous avons fait un calcul analytique en conservant les mêmes équations que dans le cas de la fermeture instantanée, sauf à remplacer $\Omega_{1}$ par $\Omega_{1}-\tau Q_{0} / 2$.

Le calcul et les constructions graphiques ont èté effectués pour des valeurs de la section utile du puits $m \mathrm{~S}=2 \mathrm{~m}^{2}$ et $m \mathrm{~S}=6 \mathrm{~m}^{2}$, avec une hauteur $h=5 \mathrm{~m}$.

La durée de fermeture a varié de zéro à $31,5 \mathrm{~s}$.

Nous reproduisons sur la figure $\mathrm{n}^{\circ} 11$ une des constructions graphiques correspondant au cas suivant :

$$
m \mathrm{~S}=6 \mathrm{~m}^{2} \quad h=5 \mathrm{~m} \quad \tau=20,60
$$

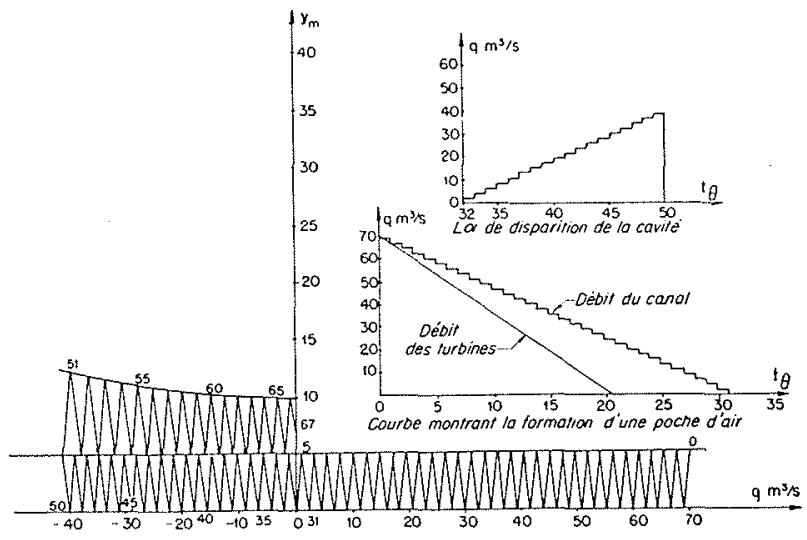

Fric. 11. - Canal de fuite comportant un puits d'aération : $h=5 \mathrm{~m} \quad m \mathrm{~S}=6 \mathrm{~m}^{2}$

Etude d'une fermeture linéaire des turbines de durée $\tau=20,6 \vartheta$.

Les tableaux suivants résument les valeurs obtenues pour la surpression par la méthode graphique et par la méthode analytique.

\begin{tabular}{|c|c|c|}
\hline & \multicolumn{2}{|c|}{$\begin{array}{c}\text { Section utile }: m \mathrm{~S}=2 \mathrm{~m} 2 \\
h=5 \mathrm{~m}\end{array}$} \\
\cline { 2 - 3 } & $\begin{array}{c}\text { Surpression } \\
\text { calculéc } \mathrm{m}\end{array}$ & $\begin{array}{c}\text { Surpression } \\
\text { déduite du } \\
\text { graphique } \mathrm{m}\end{array}$ \\
\hline & & \\
& 67,60 & 49 \\
& & \\
$5,70=6,84 \mathrm{~s}$ & 56,10 & 44,40 \\
$10,20=12,20 \mathrm{~s}$ & 46,00 & 38,50 \\
$15,40=18,50 \mathrm{~s}$ & 37,20 & 31,00 \\
$19,4 \theta=23,30 \mathrm{~s}$ & 26,60 & 23,40 \\
$26,60=31,90 \mathrm{~s}$ & 14,80 & 14,50 \\
& & \\
\hline
\end{tabular}

\begin{tabular}{|c|c|c|}
\hline & \multicolumn{2}{|c|}{$\begin{array}{c}\text { Section utile }: m \mathrm{~s}=6 \mathrm{~m}^{2} \\
h=5 \mathrm{~m}\end{array}$} \\
\cline { 2 - 3 } & & \multicolumn{2}{|c|}{$\begin{array}{c}\text { Surpression } \\
\text { Durée de fermeture }\end{array}$} & $\begin{array}{c}\text { Surpression } \\
\text { calculée } \mathrm{m}\end{array}$ & $\begin{array}{c}\text { dédu } \\
\text { graphique m }\end{array}$ \\
& & \\
\hline & 11,95 & 11,4 \\
$5,7 \theta=6,84 \mathrm{~s}$ & 10,7 & 10,7 \\
$10,7 \theta=12,80 \mathrm{~s}$ & 9,6 & 9,6 \\
$15,2 \theta=18,20 \mathrm{~s}$ & 8,6 & 8,6 \\
$19,3 \theta=23,20 \mathrm{~s}$ & 7,7 & 7,7 \\
$20,60=24,70 \mathrm{~s}$ & 7,4 & 7,4 \\
$26,6 \theta=31,90 \mathrm{~s}$ & 6,0 & 6,0 \\
& & \\
\hline
\end{tabular}

Les différences observées dans le cas de la section utile $m \mathrm{~S}=\mathbf{2} \mathrm{m}^{2}$ s'expliquent comme nous l'avons précisé pour l'étude d'une fermeture instantanée. 


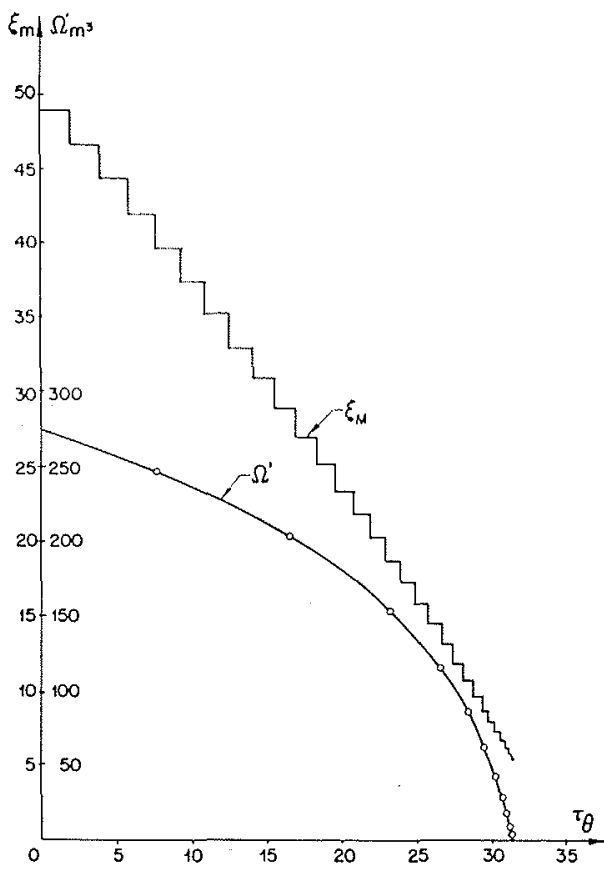

Fis. 12. - Courbes de surpression et du volume déversé en fonction du temps de fermeture des turbines: $h=5 \mathrm{~m} \quad m \mathrm{~S}=2 \mathrm{~m}^{2}$

Les courbes des figures 12 et 13 reproduisent les variations de la surpression et du rolume d'eau déversé en fonction de la durée de fermeture des turbines.

La surpression varie notablement dans le cas d'une faible section du puits. La valeur de la pression lotale $y_{2}$ passe de $54 \mathrm{~m}$ a $10 \mathrm{~m}$ pour une durée de fermeture du distributeur qui croil de zéro à $37,80 \mathrm{~s}(31,50)$.

La relation permellant le calcul du volume déversé est, dans le cas d'une fermeture linéaire,

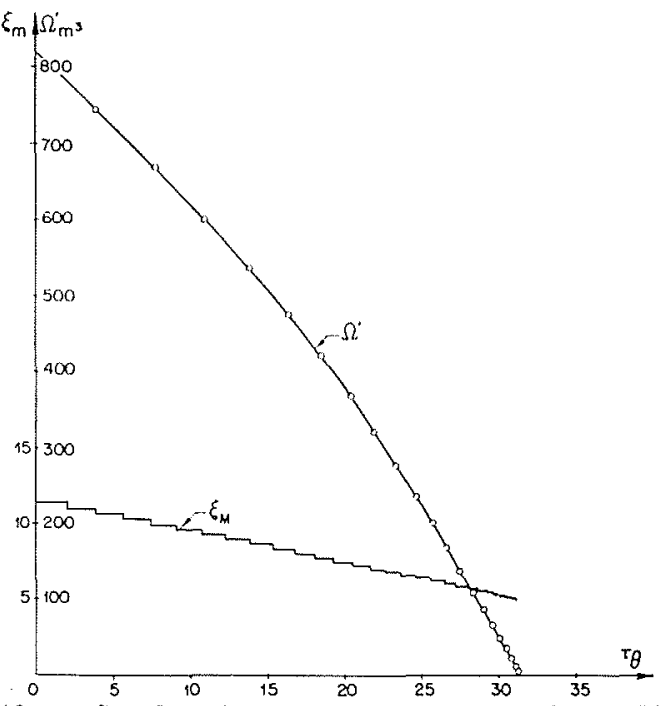

Fig. 13. - Courbes de surpression et du volume déversé en fonction du temps de fermeture des turbines: $h=5 \mathrm{~m} \quad m \mathrm{~S}=6 \mathrm{~m}^{2}$

identique a la relation utilisée pour une fermeture instantanée.

$$
\Omega^{\prime}=\frac{\mathrm{L} m^{2} \mathrm{~S}^{2}}{f} \log \left[1+\frac{f^{2}}{2 g h m^{2} \mathrm{~S}^{2}} W_{2^{2}}^{2}\right]
$$

Seule, la valeur de $\mathrm{W}_{2}$ a varié:

$$
W_{2}=W_{0} \sqrt{1-\frac{g y_{0}}{\mathrm{I}, W_{0}}}
$$

Pour le cas dont la construction graphique est reproduite sur la figure 10 , le volume calculé est égal à $377 \mathrm{~m}^{3}$ contre $362 \mathrm{~m}^{*}$ déduit de la courbe de la figure 13 (section utile $m \mathrm{~S}=6 \mathrm{~m}^{2}$ ct durée de fermeture $\tau=20,60$ ).

\section{CONCLUSION}

On voit que, dans le cas de fermetures relativement rapides par rapport à la durée d'un aller et retour d'onde dans le canal de fuite, des surpressions notables peuvent prendre naissance dans cet ouvrage.

En l'absence de tout dispositif de protection, le phénomène de savitation peut domner à ces surpressions une valeur inadmissible.

Par contre, le dispositif de clapet suggéré par M. le Président A. Canrotor roduit considérablement la surpression.

De même, si on place une cheminée d'aération fonctionnant en cheminée déversante lors du re- foulement de l'eau, on peut obtenir une action des plus favorables sur lés coups de bélier : toutelois, si on est obligé de prévoir l'accumulation de l'eau déversée par la cheminée, on peut être conduit à la constitution d'un réservoir pour lequel les dépenses seraient comparables à la solution chambre d'équilibre.

Donc, dans la mesure où on peut accepter le risque que comporte le fonctionnement de toul appareil mécanique, le clapet constitue la solution la plus économiqu'e. Si l'on veut éviter ce risque, l'adoption d'une chambre d'équilibre, conformément aux méthodes classiques, se trouve entièrement justifiée. 


\title{
ETUDE THÉORIQUE \\ DU FONCTIONNEMENT EN CHARGE \\ DES CANAUX DE FUITE \\ EN L'ABSENCE DE CHEMINEE D'EQUILIBRE
}

\author{
(Cas oú l'on thent compte des pertes de charge)
}

Nous allons reprendre les calculs exposés au chapitre précédent, mais en tenant compte, maintenant, de la perte de charge dans le canal de fuite, de la forme:

$$
\mathrm{P}=b^{2} \mathrm{~W}^{2}
$$

en utilisant uniquement la méthode analytique.

Nous étudierons, tout d'abord, les mancuvres de fermeture instantanées, en distinguanl successivement les trois cas précedemment envisagés :

-. Absence de tout dispositif d'aération.

-.- Clapet en tête du canal.

- Puis d'aćration.

Nous examinerons ensuite le cas des fermetures linéaires.

\section{CANAL DE FUITE NE COMPORTANT AUCUNE POSSIBILITE D'AERATION FERMETURE INSTANTANEE}

L'écfuation des forces vives s'écrit maintenant :

$$
\frac{\mathrm{L}}{g} \frac{d \mathrm{~W}}{d t}+y_{0}+y_{t t}+\mathrm{P}=0
$$

et nous posons :

$$
y_{0}+y_{a}=\mathrm{C}^{2}
$$

avec :

$$
\mathrm{P}=b^{2} \mathrm{~W}^{2}
$$

L’équation précédente devient:

$$
\frac{\mathrm{L}}{g} \frac{d \mathrm{~W}}{d t}+\mathrm{C}^{2}+b^{2} \mathrm{~W}^{2}=0
$$

ou :

$$
-\frac{g}{\mathrm{~L}} d t=\frac{d \mathrm{~W}}{\mathrm{C}^{2}+b^{2} \mathrm{~W}^{2}}=\frac{1}{b} \frac{d(b \mathrm{~W})}{\mathrm{C}^{2}+b^{2} \mathrm{~W}^{2}}
$$

d'où :

$$
-\frac{g}{\mathrm{~L}} t=\frac{1}{\mathrm{C} b} \operatorname{Arctg}\left(\frac{b}{\mathrm{C}} \mathrm{W}\right)+\lambda
$$

ou, compte tenu des conditions initiales : $t=0, \quad \mathrm{~W}=0$.

$t=\frac{\mathrm{L}}{g \mathrm{C} b}\left\lceil\operatorname{Arctg} \frac{b}{\mathrm{C}} \mathrm{W}_{0}-\operatorname{Arctg} \frac{b}{\mathrm{C}} \mathrm{W}\right]$

On en déduit :

$$
\mathrm{W}=\frac{\mathrm{C}}{b} \times \frac{\frac{b}{\mathrm{C}} \mathrm{W}_{0}-\operatorname{tg} \frac{g \mathrm{C} b}{\mathrm{~L}} t}{1+\frac{b}{\mathrm{C}} \mathrm{W}_{0} \operatorname{tg} \frac{g \mathrm{C} b}{\mathrm{~L}} t}
$$

Le volume de la cavité augmente jusqu'au moment $t_{1}$ où $\mathrm{W}$ s'annule :

$$
t_{1}=\frac{\mathrm{L}}{g \mathrm{C} b} \operatorname{Arctg}\left[\frac{b}{\mathrm{C}} \mathrm{W}_{0}\right]
$$

Le volume de la cavité est alors maximum et égal à $\Omega_{1}$.

$$
\Omega_{1}=f \int_{0}^{t_{1}} \mathrm{~W} d t=f \frac{\mathrm{C}}{b} \int_{0}^{t_{1}} \frac{\frac{b}{\mathrm{C}} \mathrm{W}_{0}-\operatorname{tg} \frac{g \mathrm{C} b}{\mathrm{~L}} t}{1+\frac{b}{\mathrm{C}} \mathrm{W}_{0} \cdot \operatorname{tg} \frac{g \mathrm{C} b}{\mathrm{~L}} t} d t
$$


ce qui peut se ramener à une intégrale de fraction rationnelle en posant :

$$
\operatorname{tg} \frac{g \mathrm{C} b}{\mathrm{~L}} t=u
$$

On peut aussi remarquer que :

$$
\int_{0}^{t_{1}} \mathrm{~W} d t=\int_{0}^{\mathrm{W}_{0}} t d \mathrm{~W}
$$

d'où :

$$
\begin{gathered}
\Omega_{1}=\frac{f \mathrm{~L}}{g \mathrm{C} b} \int_{0}^{\mathrm{W}_{0}}\left[\operatorname{Arctg} \frac{b}{\mathrm{C}} \mathrm{W}_{0}-\operatorname{Arctg} \frac{b}{\mathrm{C}} \mathrm{W}\right] d \mathrm{~W} \\
\Omega_{1}=\frac{f \mathrm{~L}}{g \mathrm{C} b} ; \mathrm{W}_{0} \operatorname{Arctg} \frac{b}{\mathrm{C}} \mathrm{W}_{0}-\mathrm{W}_{0} \operatorname{Arctg} \frac{b}{\mathrm{C}} \mathrm{W}_{0} \\
\left.\quad+1 / 2 \times \frac{\mathrm{C}}{b} \log \left(1+\frac{b^{2}}{\mathrm{C}^{2}} \mathrm{~W}_{0}^{2}\right)\right\} \\
\Omega_{1}=\frac{f \mathrm{~L}}{2 g b^{2}} \log \left(1+\frac{b^{2}}{\mathrm{C}^{2}} \mathrm{~W}_{0}^{2}\right)
\end{gathered}
$$

La cavité commence alors à se refermer et l'on a, la vitesse $W$ devenant négative :

$$
\frac{\mathrm{L}}{g} \frac{d \mathrm{~W}}{d t}+y_{0}+y_{a}-\mathrm{P}=0
$$

ou :

$$
\frac{\mathrm{L}}{g} \frac{d \mathrm{~W}}{d t}+\mathrm{C}^{2}-b^{2} \mathrm{~W}^{2}=0
$$

d'où :

$$
t=-\frac{\mathrm{L}}{g b} \int \frac{d(b \mathrm{~W})}{\mathrm{C}^{2}-(b \mathrm{~W})^{2}}+\lambda
$$

et :

$$
t=\frac{-\mathrm{L}}{2 g \mathrm{C} b} \log \frac{\mathrm{C}+b \mathrm{~W}}{\mathrm{C}-b \mathrm{~W}}+\lambda
$$

Pour $t=t_{1}$, on a : $\mathrm{W}=0$, d'où :

$$
t=t_{1}+\frac{\mathrm{L}}{2 g \mathrm{C} b} \log \frac{\mathrm{C}-b \mathrm{~W}}{\mathrm{C}+b \mathrm{~W}}
$$

On en déduit :

$$
\frac{\mathrm{C}-b \mathrm{~W}}{\mathrm{C}+b \mathrm{~W}}=e^{\frac{2 g \mathrm{C} b}{\mathrm{~L}}\left(t-t_{1}\right)}
$$

d'où :

$$
\mathrm{W}=\frac{\mathrm{C}}{b} \frac{1-e^{\frac{2 g \mathrm{C} b}{\mathrm{~L}}\left(t-t_{1}\right)}}{1+e^{\frac{2 g \mathrm{C} b}{\mathrm{~L}}\left(t-t_{1}\right)}}
$$

La cavité achève de se refermer à l'instant $t_{2}$, tel que :

$$
f \int_{t_{1}}^{t_{2}}|\mathrm{~W}| d t=\Omega_{1}
$$

ou :

$$
\int_{t_{1}}^{t_{2}} \frac{1-e^{\frac{2 g \mathrm{C} b}{\mathrm{~L}}\left(t-t_{1}\right)}}{1+e^{\frac{2 g \mathrm{C} b}{\mathrm{~L}}\left(t-t_{1}\right)}} d t=\frac{b}{\mathrm{C} f} \Omega_{1}
$$

ce qui peut se ramener à une intégrale de fraction rationnelle en posant :

$$
e^{\frac{2 g \mathrm{C} b}{\mathrm{~L}}\left(t-t_{1}\right)}=u
$$

On peut aussi écrire :

$$
\left|\int_{t_{1}}^{t_{2}} \mathrm{~W} d t\right|=\left|t_{2} \mathrm{~W}_{2}-\int_{0}^{\mathrm{W}_{2}} t d \mathrm{~W}\right|
$$

l'égalité précédente devient alors :

$\left|t_{2} \mathrm{~W}_{2}-\int_{0}^{\mathrm{W}_{2}}\left[t_{1}+\frac{\mathrm{L}}{2 g \mathrm{C} b} \log \frac{\mathrm{C}-b \mathrm{~W}}{\mathrm{C}+b \mathrm{~W}}\right] d \mathrm{~W}\right|=\frac{\varrho_{1}}{f}$

Soit encore :

$$
\mid\left(t_{2}-t_{1}\right) \mathrm{W}_{2}-\frac{\mathrm{L}}{2 g \mathrm{C} b^{2}}
$$

$\left\{b \mathrm{~W}_{2} \log \frac{\mathrm{C}-b \mathrm{~W}_{2}}{\mathrm{C}+b \mathrm{~W}_{2}}-a \log \frac{\mathrm{C}^{2}-b^{2} \mathrm{~W}^{2}}{\mathrm{C}^{2}}\right\}=\frac{\Omega_{1}}{f}$

Mais :

$$
t_{2}-t_{1}=\frac{\mathrm{L}}{2 g \mathrm{C} b} \log \frac{\mathrm{C}-b \mathrm{~W}_{2}}{\mathrm{C}+b \mathrm{~W}_{2}}
$$

On a donc :

$$
\left|\log \frac{\mathrm{C}^{2}-b^{2} \mathrm{~W}^{2}}{\mathrm{C}^{2}}\right|=\frac{2 g b^{2} \Omega_{1}}{\mathrm{~L} f}
$$

D'où :

$$
\mathrm{W}_{2}=\frac{\mathrm{C}}{b} \sqrt{\frac{e^{\frac{2 q b^{2} \Omega_{1}}{\mathrm{~L} f}}-1}{e^{\frac{2 g b^{2} \Omega_{1}}{\mathrm{~L} f}}}}
$$

Mais nous avons vu que:

$$
\frac{2 g b^{2}}{\mathrm{~L} f} \Omega_{1}=\log \left(1+\frac{b_{2}}{\mathrm{C}^{2}} \quad \mathrm{~W}_{0}^{2}\right)
$$


Il en résulte que :

$$
W_{2}=\frac{\mathrm{C}_{1}}{b} \sqrt{\frac{e^{\log \left[1+\left(b^{2} / \mathrm{C}^{2}\right) \mathrm{Wo}_{0}^{2}\right]}-1}{\left.e^{\log \left[1+\left(b^{2} / \mathrm{C}^{2}\right)\right.}-\mathrm{W}_{0^{2}}\right]}}
$$

$$
=\frac{\mathrm{C}}{b} \sqrt{\frac{1+\frac{b^{2}}{\mathrm{C}^{2}} \mathrm{~W}_{0}{ }^{2}-1}{1+\frac{b^{2}}{\mathrm{C}^{2}} \mathrm{~W}_{0}{ }^{2}}}=\sqrt{\frac{\mathrm{W}_{0}{ }^{2}}{1+\frac{b^{2}}{\mathrm{C}^{2}} \mathrm{~W}_{0}{ }^{2}}}
$$

Mais :

$$
b^{2}=\frac{\mathrm{P}}{\mathrm{W}^{2}}=\frac{\mathrm{P}_{0}}{\mathrm{~W}_{0}{ }^{2}}
$$

$P_{0}$ étant la perte de charge correspondant au débit initial, et $\mathrm{C}^{2}=y_{0}+y_{a}$.

On peut done écrire :

$$
\mathrm{W}_{2}=\sqrt{\frac{\mathrm{W}_{0}{ }^{2}}{1+\frac{\mathrm{P}_{0}}{\mathrm{~W}_{0}{ }^{2}} \times \frac{\mathrm{W}_{0}{ }^{2}}{y_{0}+\eta_{n}}}}
$$

oul :

$$
W_{2}=\frac{W_{0}}{\sqrt{1+\frac{\mathrm{P}_{0}}{y_{0}+y_{a}}}}
$$

On en déduit la surpression dans le canal :

$$
\xi_{\mathrm{M}}=\frac{a \mathrm{~W}_{2}}{g}=\frac{a \mathrm{~W}_{0}}{g} \times \frac{1}{\sqrt{1+\frac{\mathrm{P}_{0}}{y_{0}+y_{11}}}}
$$

a étant la célérité pour le canal étudié.

Nous avons appliqué les formules précédentes à l'exemple cité dans le chapitre précédent, en considérant deux valeurs différentes de la perte

\begin{tabular}{|c|c|c|c|}
\hline $\begin{array}{l}P_{0} \\
m\end{array}$ & $\begin{array}{c}t_{1} \\
\mathrm{~s}\end{array}$ & $\begin{array}{c}\Omega_{1} \\
\mathrm{~m}^{3}\end{array}$ & $\frac{\xi_{\mathrm{M}}}{\mathrm{m}}$ \\
\hline 0 & 12,62 & 444 & 315 \\
\hline 0,52 & 12,44 & 436,2 & 311 \\
\hline 0,78 & 12,43 & 432,37 & 308,6 \\
\hline
\end{tabular}
de charge $\mathrm{P}_{0}$ correspondant au débit maximum :

$$
0,52 \mathrm{~m} \quad 0,78 \mathrm{~m}
$$

Le tableau suivant, complété par les valeurs déjà obtenues en négligeant la perte de charge, donne les résultats obtenus :

On voit que la perte de charge ne réduit la surpression que dians une proportion infime.

\section{CANAL DE FUITE COMPORTANT UN CLAPET FERMETURE INSTANTANEE}

(ETUDE TENANT COMPTE DRS PERTES DE CHARGE)

L'écluation des forces vives s’écrit maintenant :

$$
\frac{\mathrm{L}}{g} \frac{d \mathrm{~W}}{d t}+y_{0}+b^{2} \mathrm{~W}^{2}=0
$$

Cette équation est rigoureusement identique, sous réserve de la substitution de $y_{0}$ à $y_{0}+y_{a}$, a l'équation que nous venons de résoudre dans l'étude de la formation de la cavité, en l'absence de tout dispositif d'aération.

Il en résulte que la poche d'air formée dans le canal atteint son volume maximum $\Omega_{1}$, à un instant $t_{1}$, donnés par les expressions suivantes :

$$
\begin{aligned}
t_{1} & =\frac{\mathrm{L}}{g \mathrm{C} b} \operatorname{Arctg}\left(\frac{b}{\mathrm{C}} \mathrm{W}_{0}\right) \\
\Omega_{1} & =\frac{l \mathrm{~L}}{2 \frac{\mathrm{g}}{2}} \log \left[1+\frac{b^{2}}{\mathrm{C}^{2}} \mathrm{~W}_{0}^{2}\right]
\end{aligned}
$$

C. ayant maintenant la valeur: $\mathrm{C}^{2}=y_{0}$.

La cavité commence alors à se refermer, $W$ derenant négatif, et l'on a :

$$
\frac{\mathrm{L}}{g} \frac{d \mathrm{~W}}{d t}+y_{0}-y-b^{2} \mathrm{~W}^{2}=0 .
$$

avee:

ou :

$$
\left(y+y_{a}\right) \Omega=y_{a} \Omega_{1}
$$

$$
y=y_{a}\left(\frac{\Omega_{1}}{\Omega}-1\right)
$$

et :

$$
\frac{d \Omega}{d l}=i \mathrm{~W}
$$

On en tire :

$$
\begin{aligned}
\frac{\mathrm{L}}{g} \frac{d \mathrm{~W}}{d t} & =\frac{\mathrm{L} \rho}{g} \mathrm{~W} \frac{d \mathrm{~W}}{d \Omega}=-y_{0}+y+b^{2} \mathrm{~W}^{2} \\
= & -y_{0}+y_{a}\left(\frac{\Omega_{1}}{\Omega}-1\right)+b^{2} \mathrm{~W}^{2}
\end{aligned}
$$

Soit :

$\frac{\mathrm{L} f}{g} \mathrm{~W} \frac{d \mathrm{~W}}{d \Omega}=-\left(y_{0}+y_{a}\right)+y_{a} \frac{\Omega_{1}}{\Omega}+b^{2} \mathrm{~W}^{2}$

$\frac{\mathrm{L} f}{2 g} \frac{d\left(\mathrm{~W}^{2}\right)}{d \Omega}=-\left(y_{0}+y_{a}\right)+y_{a} \frac{\Omega_{1}}{\Omega}+b^{2} \mathrm{~W}^{2}$ 
En posant $W^{2}=\alpha$, on obtient l'équation différentielle :

$$
\frac{\mathrm{L} f}{2 g} \frac{d \alpha}{d \Omega}=--\left(y_{\mathrm{a}}+y_{a}\right)+y_{a} \frac{\Omega_{1}}{\Omega}+b^{2} \alpha
$$

dont la résolution par les méthodes habituelles conduit à une intégrale de la forme :

$$
\int \frac{e^{x} d x}{x}
$$

qui ne peut être intégrée qque par l'intermédiaire d'un développement en série.

Nous avons préféré recourir à une méthode de résolution semi-graphique de l'équation différentielle, méthode inspirée des procédés utilisés dans l'étude des chambres d'équilibre et basée sur la considération des centres de courbure successifs de la courbe $(\Omega$, r).

On part de la valeur de :

$\frac{d \alpha}{d \Omega}=-\frac{2 g}{\mathrm{~L} f}\left(y_{0}+y_{a}\right)+\frac{2 g \Omega_{1} y_{a}}{\mathrm{~L} f} \frac{1}{\Omega}+\frac{2 g b^{2} \alpha}{\mathrm{L} f}$

expression de la forme :

$$
\frac{d \alpha}{d \Omega}=-\cdot \mathrm{A}+\frac{\mathrm{B}}{\Omega}+\mathrm{C} \alpha
$$

avec les conditions initiales :

$$
\alpha=0 \text { et } \Omega=\Omega_{1},
$$

ce qui donne pour valeur de la pente de la tangente au départ de la courbe $(\Omega, \alpha)$ :

$$
\left(\frac{d \alpha}{d \varrho}\right)_{1}=-A+\frac{B}{\Omega_{1}}
$$

La pente de la normale, en un point queleonque de la courbe, est :

$$
\frac{-1}{\frac{d \alpha}{d \Omega}}=\frac{1}{\left(\mathrm{~A}-\frac{\mathrm{B}}{\Omega}\right)-\mathrm{C} \alpha}
$$

Le rayon de courbure initial est donné par l'expression :

$$
i_{1}=\frac{\left[1+\left(\frac{d \alpha}{d \Omega}\right)_{1}^{2}\right]^{3 / 2}}{\left|\left(\frac{d^{2} \alpha}{d \Omega^{2}}\right)_{1}\right|}
$$

$\left(\frac{d^{2} \alpha}{d \Omega^{2}}\right)_{1}$ désignant'la valeur initiale de :

$$
\frac{d^{2} \alpha}{d \Omega^{2}}=-\frac{\mathrm{B}}{\Omega^{2}}+\mathrm{C} \frac{d \alpha}{d \Omega}
$$

Connaissant le point initial $\mathrm{M}_{1}\left(\Omega_{1}, 0\right)$ de la courbe, ainsi que là tangente et le rayon de cour- bure en ce point, on trace la portion du cercle de courbure voisine de $\mathrm{M}_{1}$, et on l'assimile à la courbe elle-même (fig. 14).

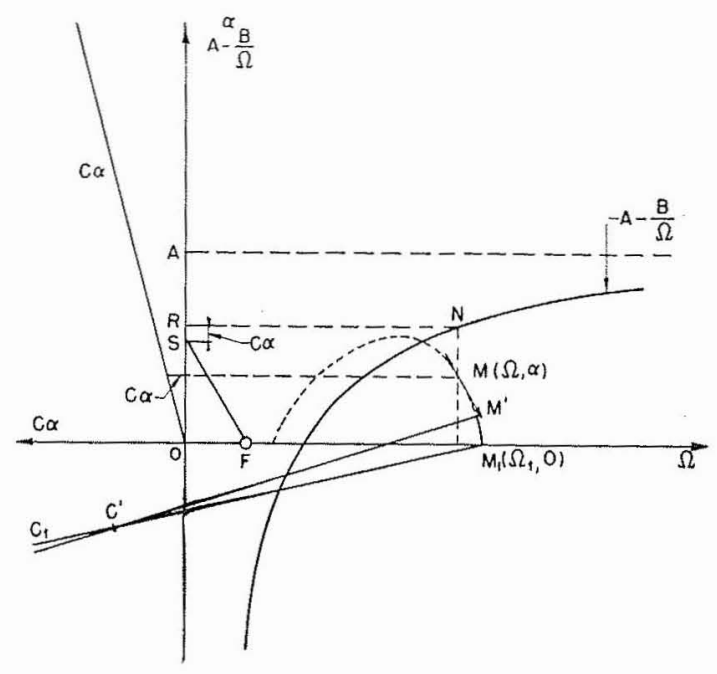

FIC. 14

La construction de la normale en un point quelconque de la courbe peut être faite de la manière suivante :

On trace l'hyperbole $H$, d'abscisse $\Omega$, d'ordonnée :

$$
A-\frac{B}{\Omega}
$$

On relève $M$ en $N$ sur la courbe $H$ et on rappelle ce point en R sur l'axe des $\alpha$; on porte alors verticalement vers le bas le segment $R S=C \approx$ : il suffit de joindre $S$ au point fixe $F$ d'abscisse égale à 1 , sur l'axe des $\Omega$, pour obtenir une parallèle $S F$ à la tangente à la courbe en M. Cette construction fournit done la direction de la normale en M.

Soit $\mathrm{M}^{\prime}$ un point voisin de $\mathrm{M}_{1}$ sur la portion initiale de la courbe : la méthode précédente donne la normale $\mathrm{M}^{\prime} \mathrm{N}^{\prime}$ en $\mathrm{M}^{\prime}$ et celle-ci coupe la normale $\mathrm{M}_{1} \mathrm{C}_{1}$ en un point $\mathrm{C}^{\prime}$ qui représente le centre de courbure en $\mathrm{M}^{\prime}$.

La construction se poursuit ainsi, de proche en proche, en assimilant chaque centre de courbure à l'intersection de deux normales consécutives.

La figure 15 donne un exemple de construction complète de la courbe $(\Omega, \alpha)$, correspondant aux données numériques de l'exemple eité dans le chapitre précédent, avec une perte de charge de $0,52 \mathrm{~cm}$ pour l'écoulement du débit maximum.

La figure 16 correspond aux mêmes données, sauf en ce qui concerne la perte de charge portée à $3,90 \mathrm{~m}$. 


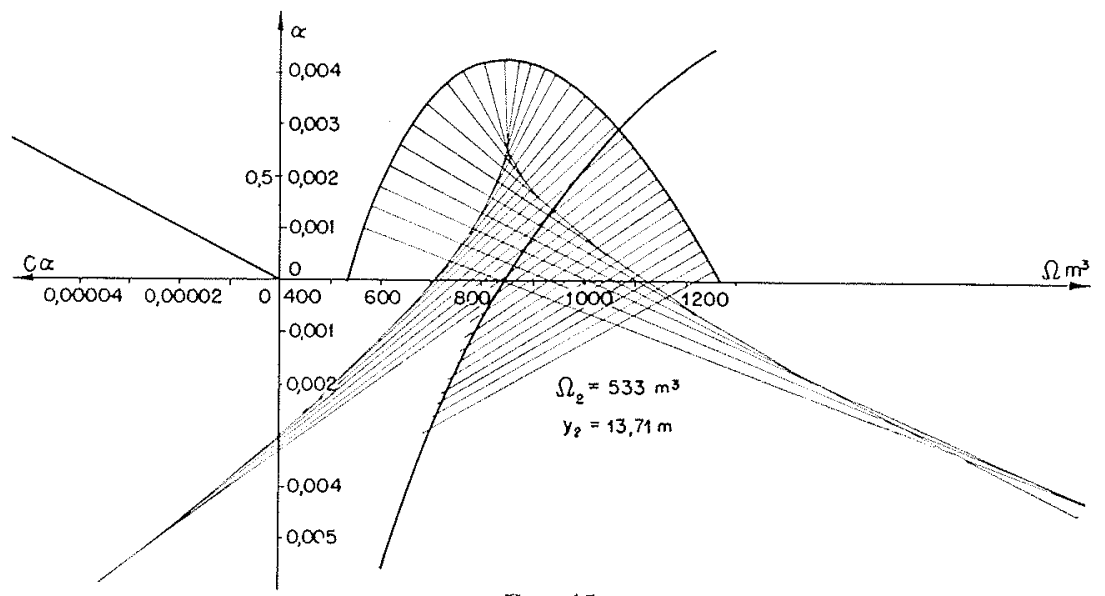

Fig, 15

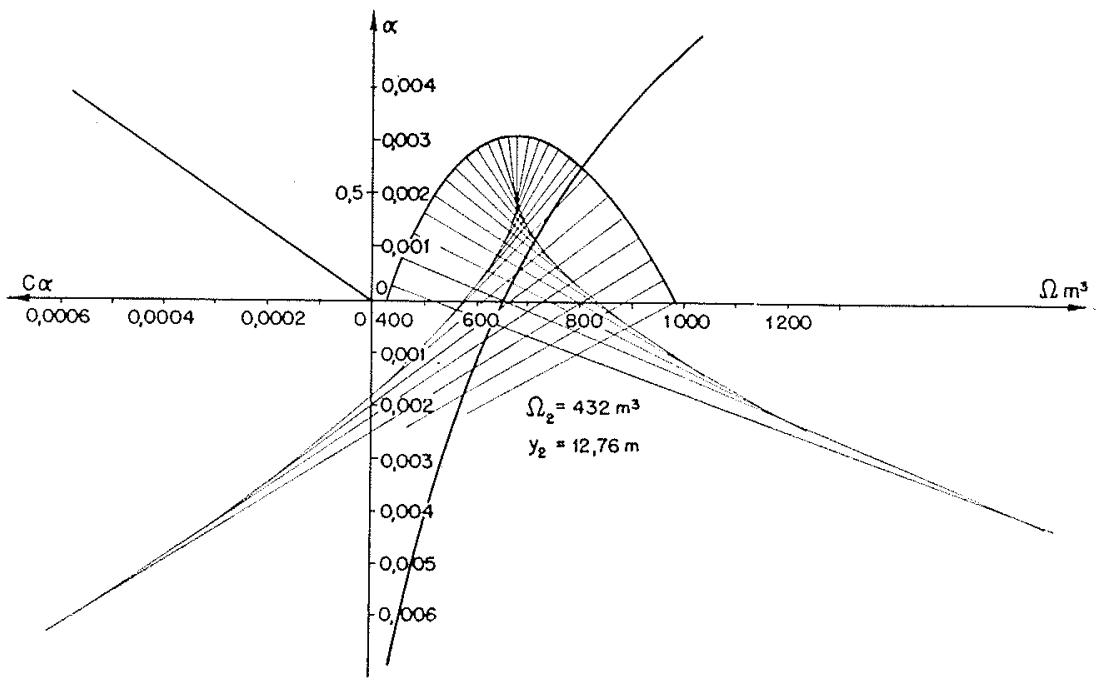

Fig. 16

Le tableau ci-dessous permet de comparer les résultats obtenus à celui qu'avait donné l'étude

\begin{tabular}{|c|c|c|c|c|}
\hline $\begin{array}{c}\mathrm{P}_{0} \\
\mathrm{~m}\end{array}$ & $\begin{array}{c}t_{1} \\
\mathrm{~s}\end{array}$ & \multicolumn{1}{|c|}{$\begin{array}{c}\Omega_{1} \\
\mathrm{~m}^{3}\end{array}$} & $\begin{array}{c}\Omega_{2} \\
\mathrm{~m}^{3}\end{array}$ & $\begin{array}{c}y_{2}=y_{0}+\xi_{\mathrm{V}} \\
(\mathrm{avec}: \\
\left.y_{0}=5 \mathrm{~m}\right)\end{array}$ \\
\cline { 1 - 4 } 0 & 38,4 & 1.332 & 551 & 14,18 \\
0,52 & 36,7 & $1.2(64$ & 533 & 13,71 \\
3,90 & 31,1 & 983 & 432 & 12,76 \\
\hline
\end{tabular}

faite au chapitre précédent en l'absence des pertes de charge. Nous reportons les valeurs de $l_{1}, \Omega_{1}$, ainsi que celles du volume $\Omega_{2}$ minimum de la poche d'air, en fin de compression, et celles de la pression maximum $y_{2}=y_{0}+\xi$ correspondante.

On voit que la perte de charge réduit la surpression, dans une proportion d'ailleurs très faible; en effet, cette réduction n'est guère que de l'ordre de $11 \%$ dans le cas de la perte de charge de $3,90 \mathrm{~m}$, qui correspond à une valeur nettement exagérée par rapport aux chiffres usuels. 


\section{CANAL DE FUITE COMPORTANT UN PUITS D'AÉRATION FERMETURE INSTANTANEE}

(ETUDE TENANT COMPTE DES PLRTES DE CHARGE)

L'équation des forces vives est identique à ceile qui correspond au cas du clapet, pendant toute la durée de la formation de la poche d'air :

$$
\frac{\mathrm{L}}{g} \frac{d \mathrm{~W}}{d t}+y_{0}+b^{2} \mathrm{~W}^{2}=0
$$

On a donc, avec $y_{0}=\mathrm{C}^{2}$ :

$$
\begin{aligned}
t_{1} & =\frac{\mathrm{L}}{g \mathrm{C} b} \operatorname{Arctg}\left(\frac{b}{\mathrm{C}} \mathrm{W}_{0}\right) \\
\Omega_{1} & =\frac{f \mathrm{~L}}{2 g b^{2}} \log \left[1+\frac{b^{2}}{\mathrm{C}^{2}} \mathrm{~W}_{0}^{2}\right]
\end{aligned}
$$

Ces valeurs sont d'ailleurs identiques à celles que l'on obtenait, en l'absence de tout dispositif d'aération, $\mathrm{C}^{2}$ étant égal maintenant à $y_{0}$ au lieu de $y_{0}+y_{a}$, valeur qu'il possédait dans ce cas.

La cavité se referme également suivant une loi identique à celle qui a été obtenue dans le cas de la cavitation, sous la même réserve de prendre $y_{0}=\mathrm{C}^{2}$. On obtient donc finalement:

$$
\begin{gathered}
\mathrm{W}_{2}=\frac{\mathrm{W}_{0}}{\sqrt{1+\frac{\mathrm{P}_{0}}{y_{0}}}} \\
t_{2}-t_{1}=\frac{\mathrm{L}}{2 g \mathrm{C} b} \log \frac{\mathrm{C}-b \mathrm{~W}_{2}}{\mathrm{C}+b \mathrm{~W}_{2}} \\
y_{\mathrm{M}}=y_{0}+\xi_{\mathrm{M}}=y_{0}+h+\frac{\mathrm{Q}_{2}{ }^{2}}{2 g \mathrm{~m}^{2} \mathrm{~S}^{2}} \\
\xi_{\mathrm{MI}}=h+\frac{f^{2} \mathrm{~W}_{2}{ }^{2}}{2 g \mathrm{~m}^{2} \mathrm{~S}^{2}}
\end{gathered}
$$

A partir de l'instant $t_{2}$ oú le déversement commence avec un débit $Q_{2}=f W_{2}$, l'équation des forces vives s'écrit :

$$
\frac{\mathrm{L}}{g} \frac{d \mathrm{~W}}{d t}-h-b^{2} \mathrm{~W}^{2}-\frac{\mathrm{Q}^{2}}{2 g m^{2} \mathrm{~S}^{2}}=0
$$

otl :

$$
\frac{\mathrm{L}}{g} \frac{d \mathrm{~W}}{d t}=h+\left\lceil b^{2}+\frac{f^{2}}{2 g m^{2} \mathrm{~S}^{2}}\right\rceil \mathrm{W}^{2}=0
$$

On en déduit :

$$
d l=\frac{\mathrm{L}}{g} \frac{d \mathrm{~W}}{h+\left[b^{2}+\frac{f^{2}}{2 g m^{2} \mathrm{~S}^{2}}\right] \mathrm{W}^{2}}
$$

d'où :

$$
t-t^{\prime}=
$$

$$
\frac{L}{g h \sqrt{\frac{b^{2}+\frac{f^{2}}{2 g m^{2} \mathrm{~S}^{2}}}{h}}} \operatorname{Arctg} \sqrt{\frac{b^{2}+\frac{f^{2}}{2 g m^{2} \mathrm{~S}^{2}}}{h}} \times \mathrm{W}
$$

$t^{\prime}$ étant une constante d'intégration.

Ceci peut s'écrire, en posant :

$$
\begin{aligned}
& \mathrm{K}=\sqrt{\frac{h}{b^{2}+\frac{\hbar^{2}}{2 g m^{2} \mathrm{~S}^{2}}}} \\
& \mathrm{~W}=\mathrm{K} \operatorname{tg}\left|\left(t-t^{\prime}\right) \frac{g h}{\mathrm{LK}}\right|
\end{aligned}
$$

A l'instant : $t_{0}=t_{1}+t_{2}$, la vitesse est. $\mathrm{W}_{2}$, d'vù :

$$
t^{\prime}=\frac{\mathrm{LK}}{g h} \operatorname{Arctg} \frac{W_{2}}{\mathrm{~K}}+t_{0}
$$

On peut done écrire :

$$
\mathrm{W}=\mathrm{K} \operatorname{tg}\left[\left(t-t_{6}\right) \frac{g h}{\mathrm{~L} \mathrm{~K}}-\operatorname{Arctg} \frac{\mathrm{W}_{2}}{\mathrm{~K}}\right]
$$

Le débit déversé à l'instant $t$ sera :

$$
q=|f \mathrm{~W}|=\left|f \mathrm{~K} \operatorname{tg}\left[\left(t-t_{0}\right) \frac{g h}{\mathrm{LK}}-\operatorname{Arctg} \frac{\mathrm{W}_{2}}{\mathrm{~K}}\right]\right|
$$

On peut calculer le volume total déversé :

$$
\Omega^{\prime}=\int_{t_{0}}^{t_{0} \pm t_{3}}|q| d t
$$

en appelant : $t_{1}+t_{2}+t_{3}$ l'instant où le déversement s'arrête.

Il vient :

$$
\Omega^{\prime}=\left|f \mathrm{~K} \int_{t_{0}}^{t_{0}+t_{3}} \operatorname{tg}\left[\left(t-t_{0}\right) \frac{g h}{\mathrm{LK}}-\operatorname{Arctg} \frac{\mathrm{W}_{2}}{\mathrm{~K}}\right] d t\right|
$$

on :

$\Omega^{\prime}=\frac{f \mathrm{~L} \mathrm{~K}^{2}}{g h}\left|\log \left\{\cos \left[\left(t-t_{0}\right) \frac{g h}{\mathrm{~L} \mathrm{~K}}-\operatorname{Arctg} \frac{\mathrm{W}_{2}}{\mathrm{~K}}\right]\right\}\right|_{t_{0}}^{t_{0} t}$ 
$t_{3}$ s'obtient en cerivant qu'à l'instant $t_{0}+t_{3}$ le débit déversé q devient nul :

$$
\left(t_{0}+t_{3}-t_{0}\right) \frac{g h}{\mathrm{~L} K}=\operatorname{Arctg} \frac{W_{2}}{\mathrm{~K}}
$$

d'où :

$$
t_{3}=\frac{\mathrm{LK}}{g h} \operatorname{Arctg} \frac{\mathrm{W}_{2}}{\mathrm{~K}}
$$

On a donc :

$$
\begin{aligned}
& \Omega^{\prime}=-\frac{f \mathrm{LK}^{2}}{g h} \log \left[\cos \left(\operatorname{Arctg} \frac{\mathrm{W}_{g}}{\mathrm{~K}}\right)\right] \\
& -\frac{f \mathrm{LK}^{2}}{g h} \log \left[\frac{\mathrm{K}}{\sqrt{\mathrm{W}_{2}^{2}+\mathrm{K}^{2}}}\right]=\frac{f \mathrm{~L} \mathrm{~K}^{2}}{2 g h} \log \left[1+\frac{\mathrm{W}_{2}^{2}}{\mathrm{~K}^{2}}\right]
\end{aligned}
$$

Il vient alors, en remplaçant $K$ par sa valeur :

$$
\Omega^{\prime}=\frac{f \mathrm{~L} \log \left\lceil 1+\left(b^{2}+\frac{f^{2}}{2 g m^{2} \mathrm{~S}^{2}}\right) \frac{\mathrm{W}_{2}^{2}}{h}\right\rceil}{2 g\left(b^{2}+\frac{f^{2}}{2 g m^{2} \mathrm{~S}^{2}}\right)}
$$

avec :

$$
b^{2}=\frac{P_{0}}{W_{0}^{2}}
$$

et :

$$
\mathrm{W}_{2}^{2}=\frac{\mathrm{W}_{0^{2}}}{1+\frac{\mathrm{P}_{0}}{y_{0}}}
$$

Nous avons appliqué ces formules à l'exemple du chapitre précédent en considérant deux vatleurs différentes de la perte de charge:

$$
0,52 \mathrm{~m} \text { et } 0,78 \mathrm{~m}
$$

\begin{tabular}{|c|c|c|c|c|}
\hline$\underset{m i}{m S}$ & $\xi_{\mathrm{M}}^{h}=0{ }_{\mathrm{m}}$ & $\begin{array}{l}h=1 \mathrm{~m} \\
\xi_{\mathrm{MI}}\end{array}$ & $\begin{array}{l}h=3 \mathrm{~m} \\
\xi_{\mathrm{NL}} \quad \mathrm{m}\end{array}$ & $\begin{array}{l}h=5 \mathrm{~m} \\
\xi_{\mathrm{NL}} \quad \mathrm{m}\end{array}$ \\
\hline 2 & $\begin{array}{l}62,4 \\
56,6 \\
54\end{array}$ & $\begin{array}{l}63,4 \\
57,6 \\
55\end{array}$ & $\begin{array}{l}65,4 \\
59,6 \\
57\end{array}$ & $\begin{array}{l}67,4 \\
61,6 \\
59\end{array}$ \\
\hline 4 & $\begin{array}{l}15,6 \\
14,15 \\
13,5\end{array}$ & $\begin{array}{l}16,6 \\
15,15 \\
14,5\end{array}$ & $\begin{array}{l}18,6 \\
17,15 \\
16,5\end{array}$ & $\begin{array}{l}20,6 \\
19,15 \\
18,5\end{array}$ \\
\hline 6 & $\begin{array}{l}6,95 \\
6,28 \\
6\end{array}$ & $\begin{array}{l}7,95 \\
7,28 \\
7\end{array}$ & $\begin{array}{l}9,95 \\
9,28 \\
9\end{array}$ & $\begin{array}{l}11,95 \\
11,28 \\
11\end{array}$ \\
\hline 8 & $\begin{array}{l}3,91 \\
3,507 \\
3,38\end{array}$ & $\begin{array}{l}4,91 \\
4,507 \\
4,38\end{array}$ & $\begin{array}{l}6,91 \\
6,507 \\
6,38\end{array}$ & $\begin{array}{l}8,91 \\
8,507 \\
8,38\end{array}$ \\
\hline
\end{tabular}

Le tableau suivant donne les valeurs de la surpression $\xi_{\text {M }}$ en fonction de $m \mathrm{~S}$ et de $h$, d'après la perte de charge : pour chaque système de valeurs de $m \mathrm{~S}$ et $h$, le tableau indique les trois valeurs de la surpression correspondant respectivement, de haut en bas, aux trois valeurs $0 \mathrm{~m}-0,52 \mathrm{~m}-0,78 \mathrm{~m}$ de $P_{0}$.
On voit que la perte de charge réduit légèrement la surpression.

Le tableau suivant donne les valeurs du volume déversé $\Omega$ en fonction de $m \mathrm{~S}$ et de $h$ d'après la perte de charge: pour chaque systime de valeurs de $m \mathrm{~S}$ et de $h$, le tableau indique les trois valeurs du volume déversé correspondant respectivement, de haut en bas, aux trois valeurs : $0 \mathrm{~m}-0,52 \mathrm{~m}-0,78 \mathrm{~m}$.

\begin{tabular}{|c|c|c|c|}
\hline $\begin{array}{c}m \mathrm{~s} \\
\mathrm{~m}^{2}\end{array}$ & $\begin{array}{c}h=1 \mathrm{~m} \\
\Omega^{\prime} \mathrm{m}^{3}\end{array}$ & $\begin{array}{c}h=3 \mathrm{~m} \\
\Omega^{\prime} \mathrm{m}^{3}\end{array}$ & $\begin{array}{c}h=5 \mathrm{~m} \\
\Omega^{\prime} \mathrm{m}^{3}\end{array}$ \\
\cline { 2 - 3 } 2 & 440 & 326 & 276 \\
& 427,5 & 315 & 265 \\
& 425 & 314 & 264 \\
4 & 1.190 & 778 & 602 \\
4 & 1.127 & 727 & 561 \\
& $1.116,5$ & 721 & 557,7 \\
& & & \\
6 & 1.970 & 1.145 & 832 \\
& 1.820 & 1.049 & 760,5 \\
& $1.784,7$ & 1.034 & 752 \\
8 & 2.700 & 1.450 & 989 \\
& 2.423 & 1.279 & 888 \\
& 2.297 & 1.232 & 862 \\
\hline
\end{tabular}

On voil que la perte de charge ne réduit le volume déversé que dans des proportions assez faibles. 


\section{INFLUENCE DE LA DUREE DE FERMETURE DES TURBINES CANAL DE FUITE NE POSSÉdANT PAS DE DISPOSITIF D'AERATION}

(MISE EN COMPTE DE LA PERTE DE CHARGE)

Nous supposons que l'arrêt du débit, au lieu d'être instantané, s'effectue linéairement en une durée $\tau$ : nous allons étudier l'influence de ce facteur, tout d'abord dans le cas où il n'existe aucun dispositf d'aération, ensuite dans le cas du clapet et dans celui du puits d'aération.

Dans les trois cas, nous ferons les mêmes hypothèses que dans le chapitre précédent, lorsque nous étudions le même probleme, sans tenir compte de la perte de charge. Autrement dit, nous admettrons, en première approximation, que le volume de la cavité ou de la bulle d'air, dans le cas de l'aération, ne diffère de celui que l'on obtient dans l'hypothèse de la fermeture instantanée que du volume total d'eau provenanl des turbines pendant la durée $\tau$, soit $Q_{0} \tau / 2$.

L'instant $t_{1}$, où le volume de la cavité est maximum, est toujours donné par :

$$
t_{1}=\frac{\mathrm{L}}{g \mathrm{C} b} \operatorname{Arctg}\left[\frac{b}{\mathrm{C}} \mathrm{W}_{0}\right]
$$

comme dans le cas de la fermeture instantanée.

Le volume maximum de la cavité est toutefois réduit de $Q_{0} \tau / 2$ par rapport à celui que l'on obtenait dans ce cas et il a donc pour valeur :

$$
\Omega_{1}=\frac{f \mathrm{~L}}{2 g b^{2}} \log \left[1+\frac{b^{2}}{\mathrm{C}^{2}} \mathrm{~W}_{0}^{2}\right]-\frac{\mathrm{Q}_{0} \tau}{2}
$$

La vitesse avec laquelle la cavité se referme est toujours :

$$
\mathrm{W}=\frac{\mathrm{C}}{b} \frac{1-e^{\frac{2 ! l \mathrm{C} b}{\mathrm{~L}}\left(t-t_{1}\right)}}{1+e^{\frac{2 g C b}{\mathrm{~L}}\left(t-t_{1}\right)}}
$$

Au moment où la fermeture est complète, elle prend la valeur $\mathrm{W}_{2}$ :

$$
\mathrm{W}_{2}=\frac{\mathrm{C}}{\bar{b}} \sqrt{\frac{e^{\frac{2 a b^{2} \xi_{1}}{\mathrm{~L} f}}-1}{e^{\frac{2 a b^{2} \Omega_{1}}{\mathrm{~L} f}}}}
$$

à condition de prendre pour $\Omega_{1}$ la nouvelle valeur obtenue, tenant compte de la durée de fermeture.

On a done :

$$
\frac{2 g b^{2} \Omega_{1}}{\mathrm{~L} f}=\log _{4}\left[1+\frac{b^{2}}{\mathrm{C}^{2}} \mathrm{~W}_{0}^{2}\right]-\frac{g b^{2} \mathrm{Q}_{0} \tau}{\mathrm{L} f}
$$

ou :

$$
\begin{gathered}
e^{\frac{2 ! b^{2} \Omega_{1}}{\mathrm{~L} f}}=e^{\log \left[1+\left(b^{2} / \mathrm{C}^{2}\right) \mathrm{W}_{0^{2}}\right]} \times e^{-\frac{\| b^{2} \mathrm{O}_{0} \tau}{\mathrm{L} f}} \\
=\left[1+\frac{b^{2}}{\mathrm{C}^{2}} \mathrm{~W}_{0^{2}}\right] \times e^{-\frac{\| b^{2} \mathrm{Q}_{0} \tau}{\mathrm{L} f}}
\end{gathered}
$$

d'où :

$$
\mathrm{W}_{2}=\frac{\mathrm{C}}{b} \sqrt{\frac{\left[1+\frac{b^{2}}{C^{2}} \mathrm{~W}_{0}^{2}\right] e^{-\frac{\| b^{2} Q_{0} \tau}{\mathrm{L} f}-1}}{\left.1+\frac{b^{2}}{C^{2}} \mathrm{~W}_{0}^{2}\right] e^{-\frac{a b^{2} Q_{0} \tau}{\mathrm{L} f}}}}
$$

oul :

$$
W_{2}=\frac{C}{b} \sqrt{\frac{1+\frac{b^{2}}{C^{2}} W_{0}^{2}-e^{\frac{g b^{2} Q_{0 \tau}}{L} f}}{1+\frac{b^{2}}{C^{2}} W_{0}^{2}}}
$$

mais :

$$
b^{2}=\frac{\mathrm{P}_{0}}{\mathrm{~W}_{0}^{2}} \quad\left(2=y_{0}+y_{a}\right.
$$

$$
\frac{\mathrm{C}}{b}=\mathrm{W}_{0} \sqrt{\frac{y_{0}+y_{a}}{\mathrm{P}_{0}}} \quad b^{2} \mathrm{~W}_{0}^{2}==\mathrm{P}_{0}
$$

$\frac{b^{2} \mathrm{~W}_{0}^{2}}{\mathrm{C}^{2}}=\frac{\mathrm{P}_{0}}{y_{0}+y_{a}} \quad \frac{g b^{2} \mathrm{Q}_{0} \tau}{\mathrm{L} j}=\frac{g b^{2} \tau \mathrm{W}_{0}}{\mathrm{~L}}=\frac{g \mathrm{P}_{0} \tau}{\mathrm{L} \mathrm{W}_{0}}$

$\mathrm{W}_{2}=\mathrm{W}_{0} \sqrt{\frac{y_{0}+y_{u}}{\mathrm{P}_{0}} \frac{1+\frac{\mathrm{P}_{0}}{y_{0}+y_{0}}-e^{\frac{g \mathrm{P}_{0}}{\mathrm{~L}_{0}}}}{1+\frac{\mathrm{P}_{0}}{y_{0}+y_{u}}}}$

$=\mathrm{W}_{0} \sqrt{\frac{y_{0}+y_{u}}{\mathrm{P}_{0}} \frac{\left(y_{0}+y_{a}\right)\left(1-e^{\left.\frac{y^{\mathrm{L}} \overline{\mathrm{W}_{0}}}{\mathrm{~W}_{0}}\right)}+\mathrm{P}_{0}\right.}{y_{0}+y_{a}+\mathrm{P}_{0}}}$

On en déduit la surpression dans le canal :

$$
\xi_{\mathrm{M}}=\frac{a \mathrm{~W}_{2}}{g}
$$

$$
=\frac{a W_{0}}{g} \sqrt{\frac{y_{0}+y_{a}}{\mathrm{P}_{0}} \frac{\left(y_{0}+y_{a}\right)\left(1-e^{\frac{g P_{0} \tau}{L} \mathrm{~W}_{0}}\right)+\mathrm{P}_{0}}{y_{0}+y_{a}+\mathrm{P}_{0}}}
$$




\section{INFLUENCE DE LA DUREE DE FERMETURE DES TURBINES CANAL DE FUITE MUNI D'UN CLAPET}

(MISE EN COMPTE DE LA PERTE DE CHARGE)

Ce calcul est rigoureusement identique à celui qui correspond au cas de la fermeture instantanée, sous réserve de tenir compte du fait que le volume maximum de la poche d'air n'est plus $a_{1}$, mais :

$$
\Omega_{1}-\frac{Q_{0} \tau}{2}
$$

\section{INFLUENCE DE LA DUREE DE FERMETURE DES TURBINES CANAL DE FUITE AVEC PUITS D'AÉRATION}

(MISE EN COMPTE DE LA PERTE DE CHARGE)

En se limitant, comme nous l'avons déjà indiqué, aux valeurs de $\tau$ relativement faibles qui correspondent aux données pratiques, on a, avec $y_{0}=\mathrm{C}^{2}$ :

$$
\begin{aligned}
t_{1} & =\frac{\mathrm{L}}{g \mathrm{C} b} \operatorname{Arctg}\left[\frac{b}{\mathrm{C}} \mathrm{W}_{0} \mid\right. \\
\Omega_{1} & =\frac{f \mathrm{~L}}{2 g b^{2}} \log \left[1+\frac{b^{2}}{\mathrm{C}^{2}} \mathrm{~W}_{0}^{2}\right]-\frac{\mathrm{Q}_{0} \tau}{2}
\end{aligned}
$$

La vilesse avec laquelle la cavité se referme est :

$$
\mathrm{W}=\frac{\mathrm{C}}{b} \frac{1-e^{\frac{2 a(: b}{\mathrm{I}}\left(t-t_{1}\right)}}{1+e^{\frac{2 a(: b}{\mathrm{L}}\left(t-t_{1}\right)}}
$$

et l'on a, au moment du début du déversement :

$$
\mathrm{W}_{2}=\frac{\mathrm{C}}{b} \sqrt{\frac{e^{\frac{2 ! l b^{2} \Omega_{1}}{\mathrm{~L} f}}-1}{e^{\frac{2 a b^{2} \Omega_{1}}{\mathrm{~L} f}}}}
$$

à condition de prendre pour $\Omega_{1}$ la valeur correspondant au cas actuel; or, on a :

$$
\begin{gathered}
\frac{2 g b^{2} \Omega_{1}}{\mathrm{~L} f}=\log \left[1+\frac{b^{2}}{\mathrm{C}^{2}} \mathrm{~W}_{1^{2}}^{2}\right]-\frac{g b^{2} \mathrm{Q}_{0} \tau}{\mathrm{L} f} \\
e^{\frac{2 a b^{2} \Omega_{1}}{\mathrm{~L} f}}=\left[1+\frac{b^{2}}{\mathrm{C}^{2}} \mathrm{~W}_{0}^{2}\right] \times e^{-\frac{g b^{2} Q_{0} \tau}{\mathrm{L} f}}
\end{gathered}
$$

C'est à partir de cette valeur initiale de $\Omega_{1}$ que doit être effectuéc la construction graphique précédemment exposée, à propos de la fermeture instantanée. d'où :

$$
\mathrm{W}_{2}=\frac{\mathrm{C}}{b} \sqrt{\frac{1+\frac{b^{2}}{\mathrm{C}^{2}} \mathrm{~W}_{0}{ }^{2}-e^{\frac{g b^{2} \mathrm{~W}_{0} \tau}{\mathrm{L}}}}{1+\frac{b^{2}}{\mathrm{C}^{2}} \mathrm{~W}_{0^{2}}^{2}}}
$$

mais :

$$
\begin{aligned}
b^{2} \mathrm{~W}_{0}^{2} & =\mathrm{P}_{0} \quad \mathrm{C}^{2}=y_{0} \quad \frac{\mathrm{C}}{b}=\mathrm{W}_{0} \sqrt{\frac{y_{0}}{\mathrm{P}_{0}}} \\
\frac{b^{2} \mathrm{~W}_{0}^{2}}{\mathrm{C}^{2}} & =\frac{\mathrm{P}_{0}}{y_{0}} \quad \frac{g b^{2} \mathrm{~W}_{0} \tau}{\mathrm{L}}=\frac{g b^{2} \mathrm{~W}_{0}^{2} \tau}{\mathrm{L} \mathrm{W_{0 }}}=\frac{g \mathrm{P}_{0} \tau}{\mathrm{L} \mathrm{W}_{0}} \\
\mathrm{~W}_{2} & =\mathrm{W}_{0} \sqrt{\frac{1+\frac{y_{01}}{\mathrm{P}_{0}}-\frac{\mathrm{P}_{0}}{1-\frac{g \mathrm{P}_{0}}{\mathrm{I} \mathrm{W}_{0}}}}{1+\frac{\mathrm{P}_{01}}{y_{0}}}}
\end{aligned}
$$

Le débit de retour traverse alors la cheminée avec une valeur initiale $f\left|\mathrm{~W}_{2}\right|$ et crée une contrepression maximum; la formule générale :

$$
y=y_{0}+h+\frac{\mathrm{Q}^{2}}{2 g m^{2} \mathrm{~S}^{2}}
$$

donne, pour la pression maximum alor's réalisée, la valeur :

$$
\begin{aligned}
y_{2} & =y_{0}+h+\frac{f^{2}\left|\mathrm{~W}_{2}\right|^{2}}{2 g m^{2} \mathrm{~S}^{2}} \\
& =y_{0}+h+\frac{f^{2} \mathrm{~W}_{0}^{2}}{2 g m^{2} \mathrm{~S}^{2}} \frac{y_{0}}{\mathrm{P}_{0}} \cdot \frac{y_{0}\left(1-e^{\left.\frac{g \mathrm{P}_{0} \tau}{\mathrm{L} \mathrm{V}_{0}}\right)+\mathrm{P}_{0}}\right.}{y_{0}+\mathrm{P}_{0}} \\
& =y_{0}+h+\frac{\mathrm{Q}_{0}{ }^{2}}{2 g m^{2} \mathrm{~S}^{2}} \frac{y_{0}}{\mathrm{P}_{0}} \frac{y_{0}\left(1-e^{\left.\frac{h \mathrm{P}_{0} \tau}{\mathrm{L} \mathrm{V}_{0}}\right)+\mathrm{P}_{0}}\right.}{y_{0}+\mathrm{P}_{0}}
\end{aligned}
$$


Postérieurement à l'instant $t_{0}=t_{1}+t_{2}$, où commence le déversement, l'équation différentielle des forces vives s'écrit exactement de la même façon que dans le cas de la fermeture instantanée, et l'on aboutit aux mêmes relations :

$$
\begin{gathered}
\mathrm{W}=\mathrm{K} \operatorname{tg}\left[\left(t-t^{\prime}\right) \frac{g h}{\mathrm{LK}}\right] \\
t-t^{\prime}=\frac{\mathrm{LK}}{g h} \operatorname{Arctg} \frac{\mathrm{W}}{\mathrm{K}}
\end{gathered}
$$

en posant :

$$
\mathrm{K}=\sqrt{\frac{h}{b^{2}+\frac{f^{2}}{2 g m^{2} \mathrm{~S}^{2}}}}
$$

et en appelant: $t^{\prime}$ une constante d'intégration, égale, puisque $\mathrm{W}=-\mathrm{W}_{2} \cdot$ à l'instant $t_{0}$, à :

$$
t^{\prime}=\frac{\mathrm{L} \mathrm{K}}{g h} \operatorname{Arctg} \frac{\mathrm{W}_{2}}{\mathrm{~K}}+t_{0}
$$

done :

$$
\mathrm{W}=\mathrm{K} \operatorname{tg}\left[\left(t-t_{0}\right) \frac{g h}{\mathrm{KL}}-\operatorname{Arctg} \frac{\mathrm{W}_{2}}{\mathrm{~K}}\right]
$$

On aboutit à la même expression du volume total déversé entre $t_{0}$ et $t_{0}+t_{3}$, instant où finit le déversement :

$\Omega^{\prime}=\frac{\rho \mathrm{L} \mathrm{K}^{2}}{g h}\left|\log \left\{\cos \left(t-t_{0}\right) \frac{g h}{\mathrm{LK}}-\operatorname{Arctg} \frac{\mathrm{W}_{2}}{\mathrm{~K}}\right\}\right|_{t_{0}}^{t_{0}+t_{3}}$

avec :

$$
t_{3}=\frac{\mathrm{L} \mathrm{K}}{g h} \operatorname{Arctg} \frac{\mathrm{W}_{2}}{\mathrm{~K}}
$$

et done :

$$
\begin{aligned}
& \Omega^{\prime}=\frac{f \mathrm{LK}^{2}}{2 g h} \log \left[1+\frac{\mathrm{W}_{2}^{2}}{\mathrm{~K}^{2}}\right] \\
& =\frac{f \mathrm{~L}}{2 g\left(b^{2}+\frac{f^{2}}{2 g m^{2} \mathrm{~S}^{2}}\right)} \log \left[1+\left(b^{2}+\frac{f^{2}}{2 g m^{2} \mathrm{~S}^{2}}\right) \frac{\mathrm{W}_{2}^{2}}{h}\right]
\end{aligned}
$$

\section{ETUDE EXPERIMENTALE}

\section{DU FONCTIONNEMENT EN CHARGE DES CANAUX DE FUITE EN L'ABSENCE DE CHEMINEES D'ÉQUILIBRE}

\section{INTRODUCTION}

Dans les deux chapitres précédents, nous avons exposé l'étude théorique du fonctionnement en charge des canaux de fuite en l'absence de cheminée d'équilibre, tout d'abord en négligeant les pertes de charge, puis en tenant compte de celles-ci.

Nous allons exposer maintenant les résultats de l'étude expérimentale, étude que nous avons faite en collaboration avec M. Michel DE ReynaL.

Nous avons étudié, d'une part, l'arrêt instantané du débit des turbines, d'autre part, la disparition progressive de celui-ci suivant une loi linéaire.
Ces études ont été faites dans trois hypothèses:

- Canal de fuite sans aucun dispositif d'aération.

- Canal muni d'un clapet (dispositif de M. CAQUOT).

- Canal muni d'un puits d'aération.

Dans nos expériences, nous nous sommes limités au cas des fermetures instantanées avec un système dont les pertes de charge n'étaient pas négligeables, en nous plaçant successivement dans les trois hypothèses ci-dessus rappelées. 


\section{CAS DU CANAL DE FUITE SANS AUCUN DISPOSITIF D'AERATION}

Le modèle étudié dont les dimensions sont indiquées sur le schéma de la figure $17 a$ et dont la photographie de la figure $17 b$ donne l'aspect général, est constitué, essentiellement, par une conduite horizontale, en tôle de $2 \mathrm{~mm}$ d'épaisseur, d'une longueur de $25 \mathrm{~m}$ et d'un diamètre

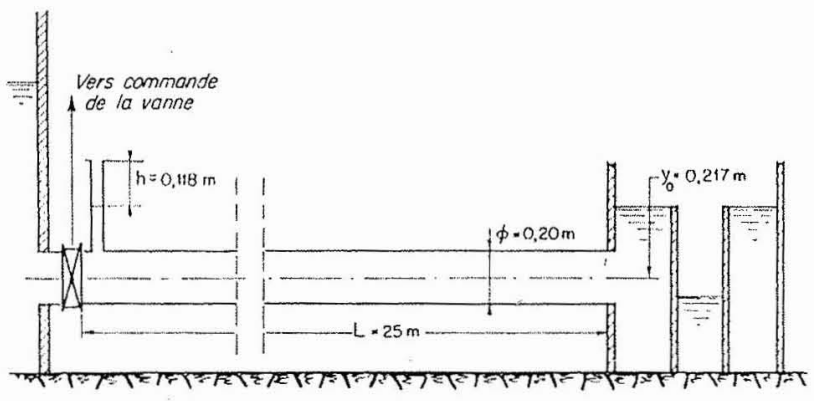

Fig. $17 a$ une durée voisine du centième de seconde grâce à la chute d'un poids tombant de $3 \mathrm{~m}$ de hauteur (fig. 18).

A l'aval, la conduite débouche dans un bassin dans lequel le niveau est maintenu pratiquement constant par un déversoir à grande longueur de seuil (fig. 19) : ce niveau est à une cote supérieure de $0,217 \mathrm{~m}$ à l'axe de la conduite.

Le débit correspondant au régime permanent initial est mesuré à l'aide d'un déversoir de BAzıN placé en série avec l'installation.

L.es pressions instantances sont enregistrées par un manometre comprenant une membrane métallique mince dont les déformations sont détectées par deux extensomètres à fil résistant : le matériel d'amplification et d'enregistrement utilisé est du type NoB-Télec.

La mesure de la perte de charge, en régime permanent, a montré que celle-ci varie, en pre-

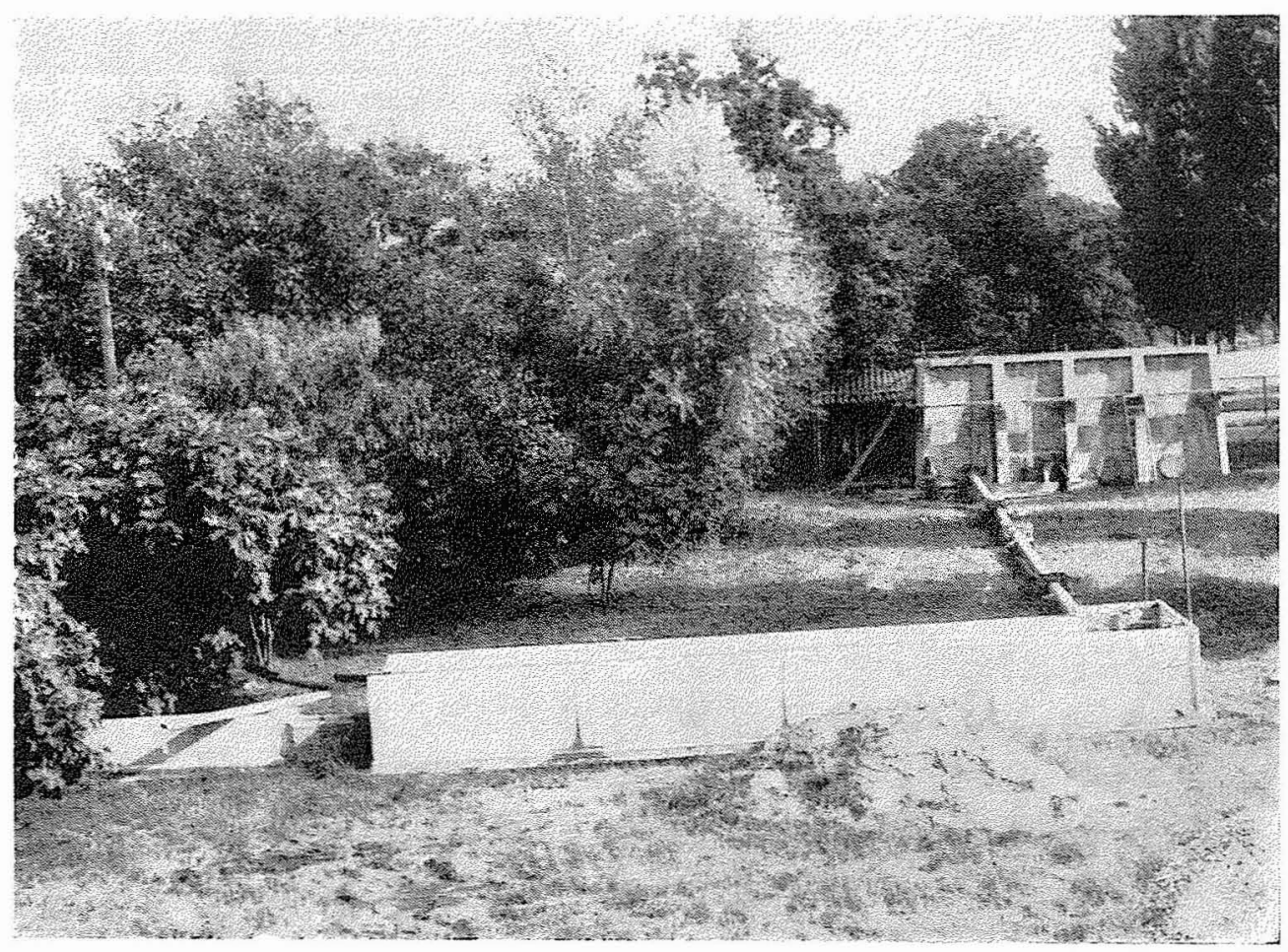

FIG. $17 b$

de $0,20 \mathrm{~m}$ : elle est formée de 4 tronçons, assemblés les uns aux autres par brides, avec interposition de joints en caoutchouc.

Cette conduite est alimentée, à son extrémité amont, par un bassin, avec lequel elle communique par l'intermédiaire d'une vanne à guillotine dont la fermeture est normalement réalisée en mière approximation, suivant une loi pratiquement quadratique, sa valeur étant de $0,038 \mathrm{~m}$ poụr un débit de $14,2 \mathrm{l} / \mathrm{s}$.

Par ailleurs, la vitesse de propagation des ondes a été déterminée par la méthode classique de la dépression brusque due à M. Camichel.

On a trouvé ainsi $a=780 \mathrm{~m} / \mathrm{s}$, valeur relati- 


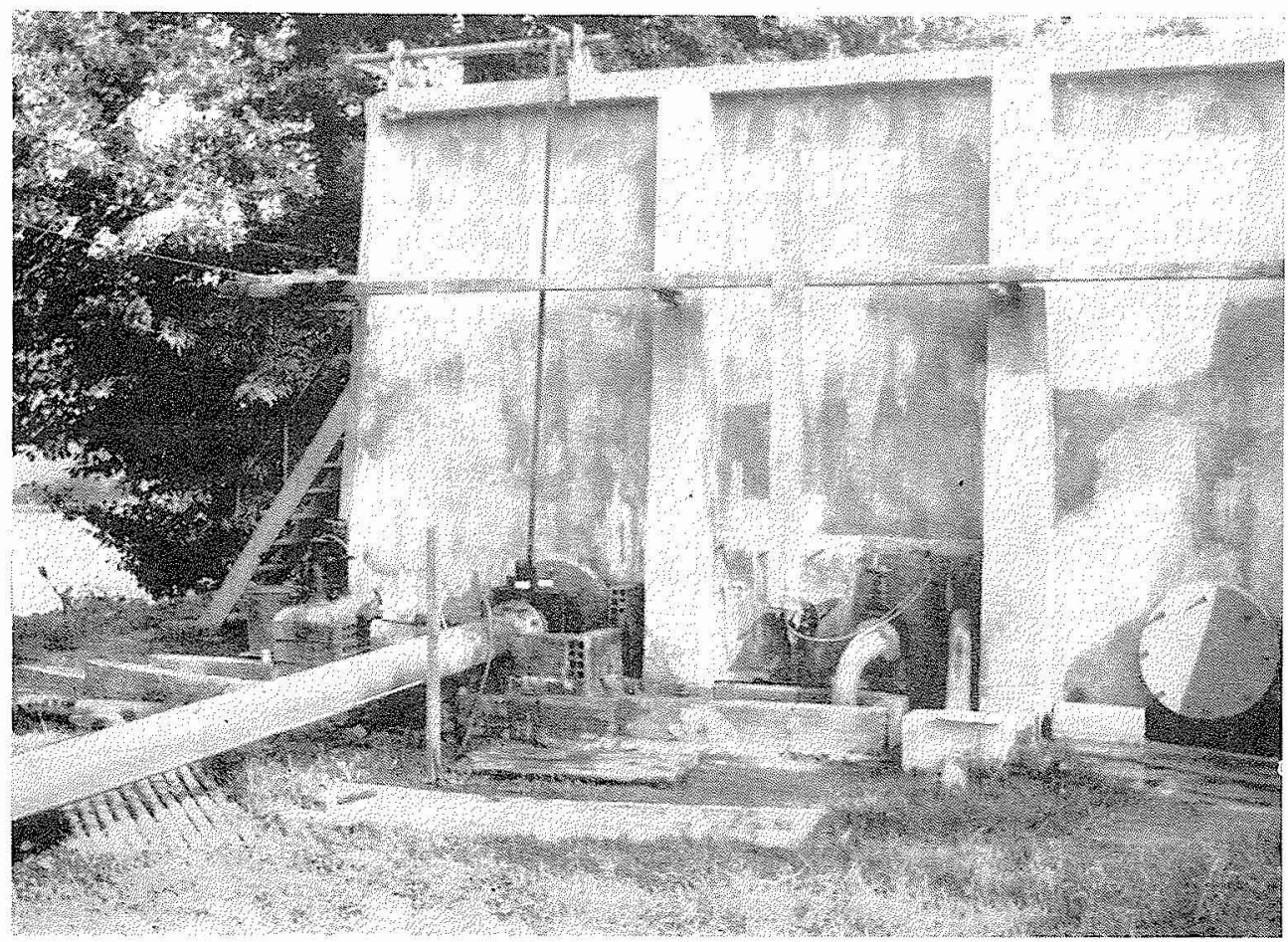

Fig. 18

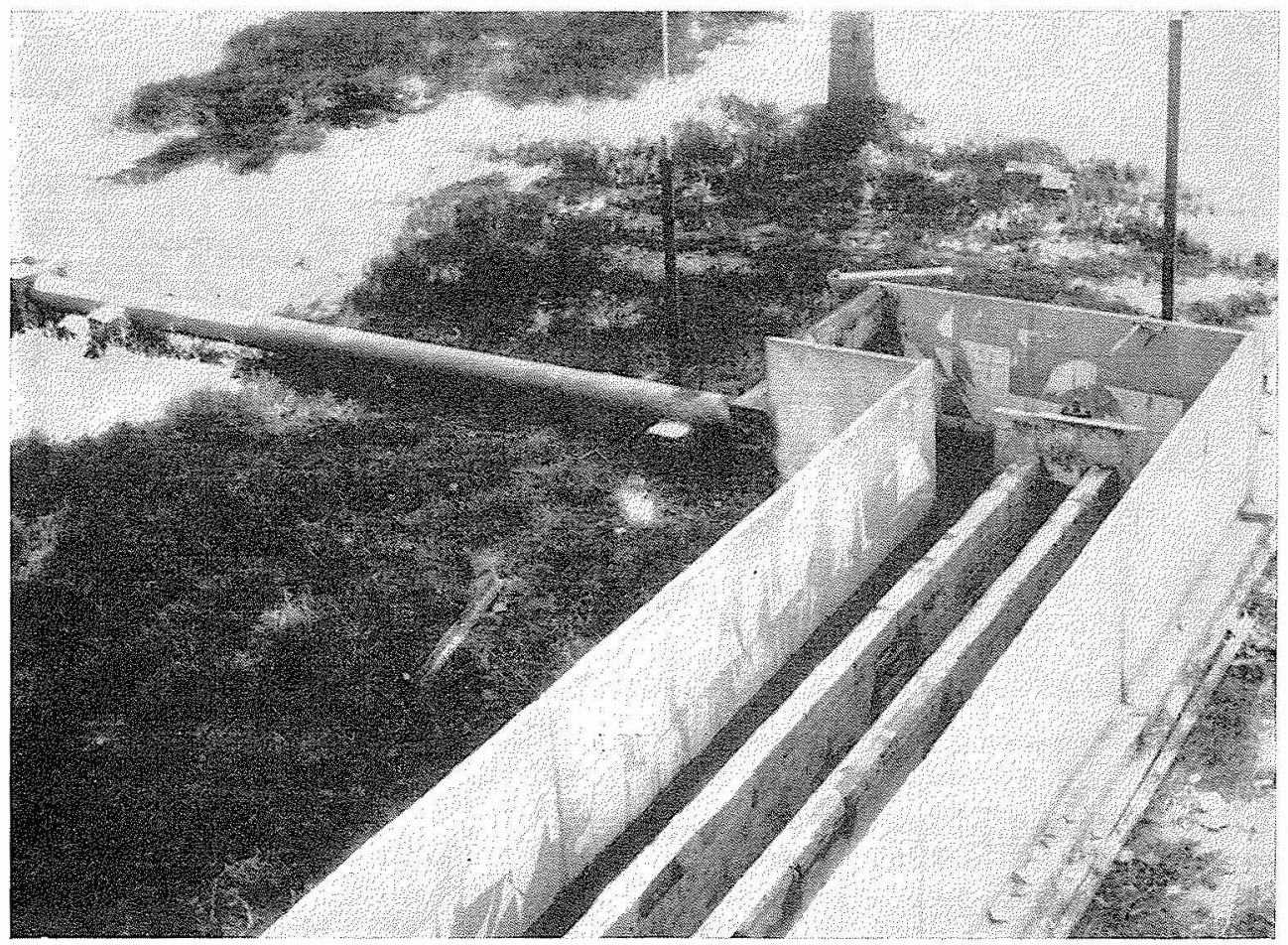

FIG, 19 


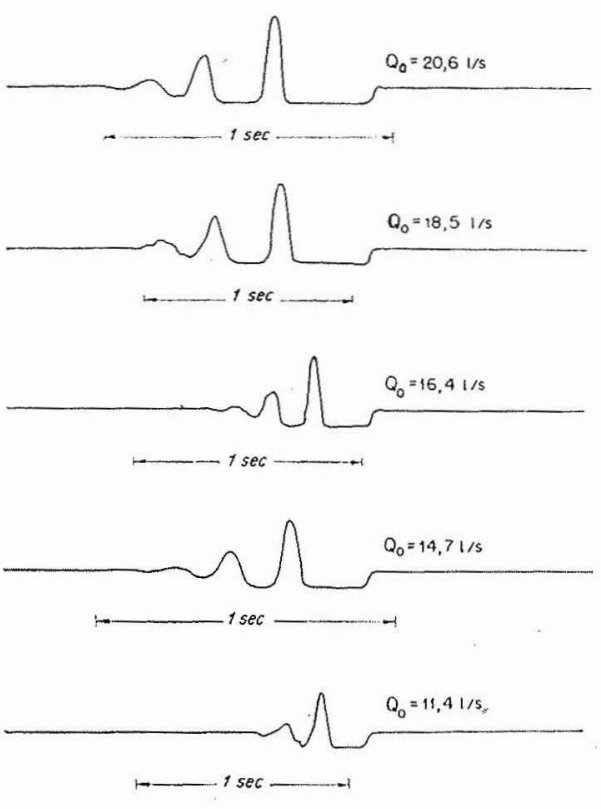

Iig. 20

TABLEAU I

\begin{tabular}{|c|c|c|c|c|}
\hline$Q_{0} l / \mathrm{s}$ & $\begin{array}{c}\xi_{\mathrm{MI}} \mathrm{m} \\
\text { expérimental }\end{array}$ & $\begin{array}{c}\xi_{\mathrm{MI}} \mathrm{m} \\
\text { théorique }\end{array}$ & $\begin{array}{c}\left(t_{1}+t_{2}\right) \mathrm{s} \\
\text { expérimental }\end{array}$ & $\begin{array}{c}\left(t_{1}+t_{2}\right) \mathrm{s} \\
\text { théorique }\end{array}$ \\
\cline { 5 - 5 } 11,4 & 26,5 & 28,8 & 0,185 & 0,178 \\
11,4 & 27,3 & 28,8 & 0,181 & 0,178 \\
12,1 & 29,3 & 30,6 & 0,183 & 0,190 \\
14,7 & 35,6 & 37,2 & 0,237 & 0,234 \\
14,7 & 36,4 & 37,2 & 0,230 & 0,234 \\
16,4 & 40,6 & 41,4 & 0,257 & 0,262 \\
16,4 & 39,3 & 41,4 & 0,254 & 0,262 \\
18,5 & 46 & 46,8 & 0,290 & 0,296 \\
18,5 & 48 & 46,8 & 0,287 & 0,296 \\
20,6 & 51,5 & 52,2 & 0,321 & 0,327 \\
20,6 & 51,5 & 52,2 & 0,310 & 0,327 \\
20,6 & 52,5 & 52,2 & 0,317 & 0,327 \\
20,6 & 51 & 52,2 & 0,321 & 0,327 \\
& & & & \\
\hline
\end{tabular}

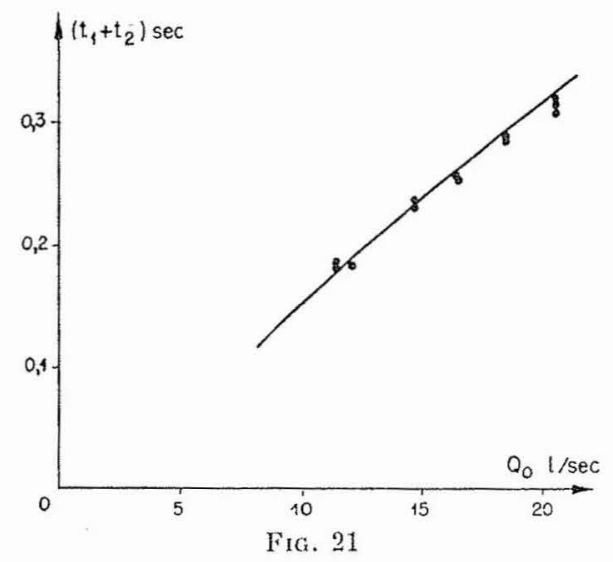

vement faible qui s'explique par la présence des joints déformables en caoutehouc.

Naturellement, chaque mesure était précédée d'une purge très soigneuse de l'air que pouvait renfermer la conduite.

Treizé mesures ont été faites, portant sur des valeurs du débit comprises entre $11,4 \mathrm{l} / \mathrm{s}$ et $20,6 \mathrm{l} / \mathrm{s}$. La figure 20 donne, à titre d'exemple, un certain nombre d'enregistrements effectués par le manomètre.

Les résultats sont fournis par le tableau I qui donne les valeurs théoriques et les valeurs expérimentales de la durée $t_{1}+t_{2}$ d'existence de la cavité, d'une part, et de la surpression maximum $\xi_{\text {XI }}$, d'autre part.

Une comparaison plus rapide entre theorie et expérience peut être faite par l'examen des courbes de la figure 21 donnant $t_{1}+t_{2}$ et de la figure 22 relative à $\zeta_{M}$. Ces courbes mettent en évidence une concordance excellente, eu égard aux hypothèses faites pour le calcul.

Il convient de remarquer que l'influence de la perte de charge est pratiquement nulle, dans ces expériences : le terme destiné à en tenir compte :

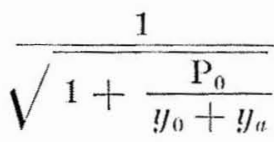

est égal à l'unité, à moins de $0,5 \%$.

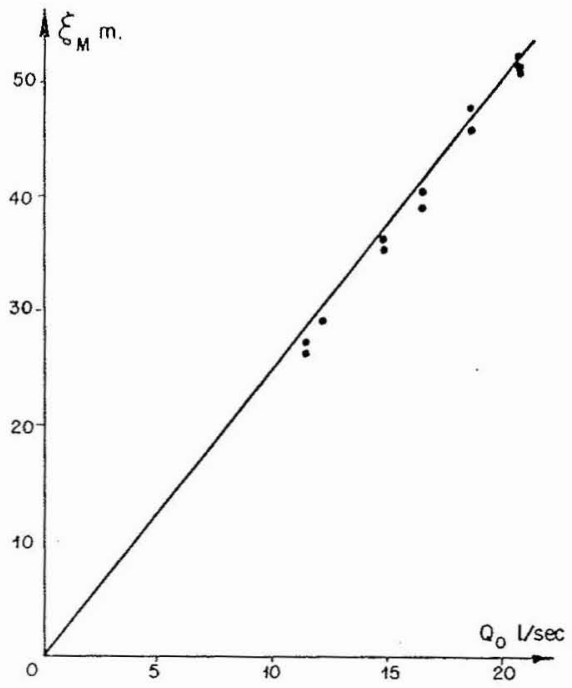

FIG. 22 


\title{
CAS DU CANAL DE FUITE MUNI D'UN CLAPET
}

\author{
(DisposituF DE M. Caquot')
}

Les expériences sont faites sur le modèle qui vient d'être décrit après avoir muni l'extrémité amont de celui-ci d'un puits d'aération qui peut être fermé par un robinet à boisseau.

Le robinet est normalement ouvert, dans le régime permanent initial et pendant la période d'aspiration d'air consécutive à la fermeture de la vanne : on observe facilement l'instant précis où s'arrête l'aspiration, le volume de la poche d'air passant par son maximum, et l'on ferme alors le robinet, de telle facon que celui-ci joue dans T'expérience le rôle d'un clapet sensible à tout écart de la pression autour de la pression atmosphérique.

Comme nous l'avons indiqué dans le chapitre précédent, le calcul du temps, $t_{2}$ ne peut pas se faire par voie analytique et la méthode graphique serait très longue.

Aussi, avons-nous fait porter seulement sur la surpression maximum $\xi_{\mathrm{M}}$ la comparaison entre théorie et expérience basée sur les neuf mesures effectuées, le débit variant de $11 \mathrm{l} / \mathrm{s}$ à $17,8 \mathrm{l} / \mathrm{s}$.

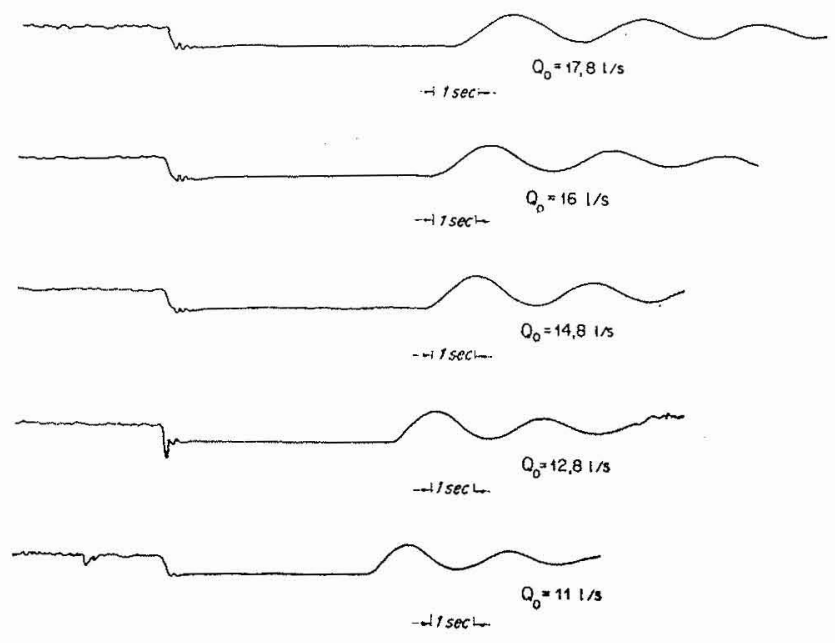

FIG. 23
La figure 23 donne, à titre d'exemple, un certain nombre d'enregistrements effectués par le manomètre.

Le tableau II et la courbe de la figure 24 montrent les résultats obtenus par le calcul et par l'expérience.

On observe la bonne concordance obtenue.

TABLEAU II

\begin{tabular}{|l|c|c|}
\hline$Q_{0} l / \mathrm{s}$ & $\begin{array}{c}\xi_{\mathrm{NI}} \mathrm{m} \\
\text { expérimental }\end{array}$ & $\begin{array}{c}\xi_{\mathrm{NI}} \mathrm{m} \\
\text { théorique }\end{array}$ \\
\hline & & \\
\hline 11 & 0,219 & 0,227 \\
11 & 0,198 & 0,227 \\
11 & 0,198 & 0,227 \\
12,8 & 0,198 & 0,229 \\
12,8 & 0,214 & 0,229 \\
14,8 & 0,240 & 0,231 \\
15,65 & 0,214 & 0,231 \\
16,7 & 0,231 & 0,2305 \\
16,8 & 0,218 & 0,2302 \\
16,8 & 0,242 & 0,2302 \\
17,3 & 0,214 & 0,229 \\
17,7 & 0,228 & 0,229 \\
17,8 & 0,238 & 0,2285 \\
17,8 & 0,230 & 0,2285 \\
& & \\
\hline
\end{tabular}

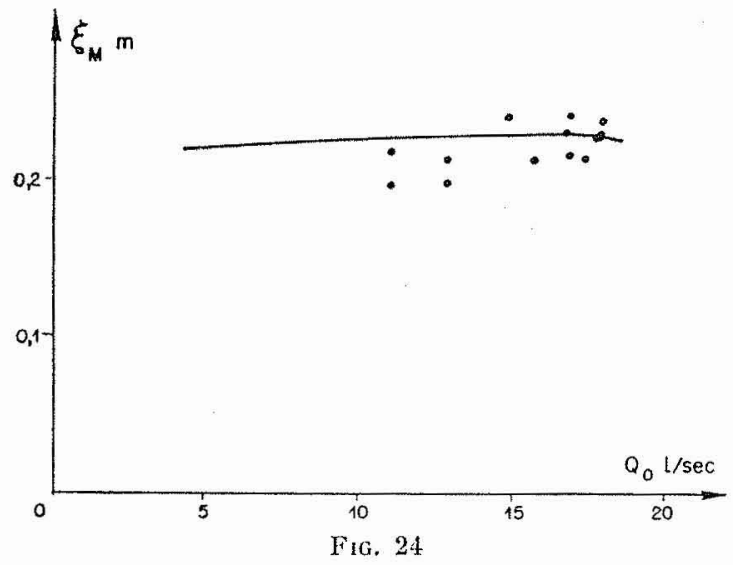

\section{CAS DU CANAL DE FUITE MUNI D'UN PUITS D'AERATION}

Le puits d'aération, visible sur la figure 25 , correspond aux valeurs:

$$
h=0,118 \mathrm{~m} \text { et } m \mathrm{~S}=0,00257 \mathrm{~m}^{2}
$$

Cette valeur de $m$ a été déterminée en fermant la vanne amont de la conduite et en ali- mentant celle-ci par l'aval avec une pression suffisante pour que l'eau puísse s'écouler par l'extrémité supérieure du puits d'aération : eu égard au fait que $S=0,003125$, la valeur de $m$ correspondante est de 0,825 . 
Indépendamment des mesures de temps of de surpression analogues à celles des cas précédents, nous avons mesuré également le volume lotal $\Omega^{\prime}$ provenant du déversement par le puits d'aćation.

La figure 26 donne quelques exemples d'enresistrement obtenus avee le manometre.

Le tableau III donne les valeurs théoriques et expérimentales de la durée $l_{1}+l_{2}$ el de la surpression maximum $\xi_{\mathrm{X}}$. $\Omega^{\prime}$

Le tableau III' est relatif au volume déversant

Les figures 27, 28, 29, donnent, en fonction du débit initial $Q_{0}$, les valeurs thériques et expérimentales de la durée $t_{1}+t_{2}$ de la surpression $\xi_{\mathrm{X}}$, et du volume déversant $\Omega^{\prime}$.

La coincidence entre théorie et expérience est

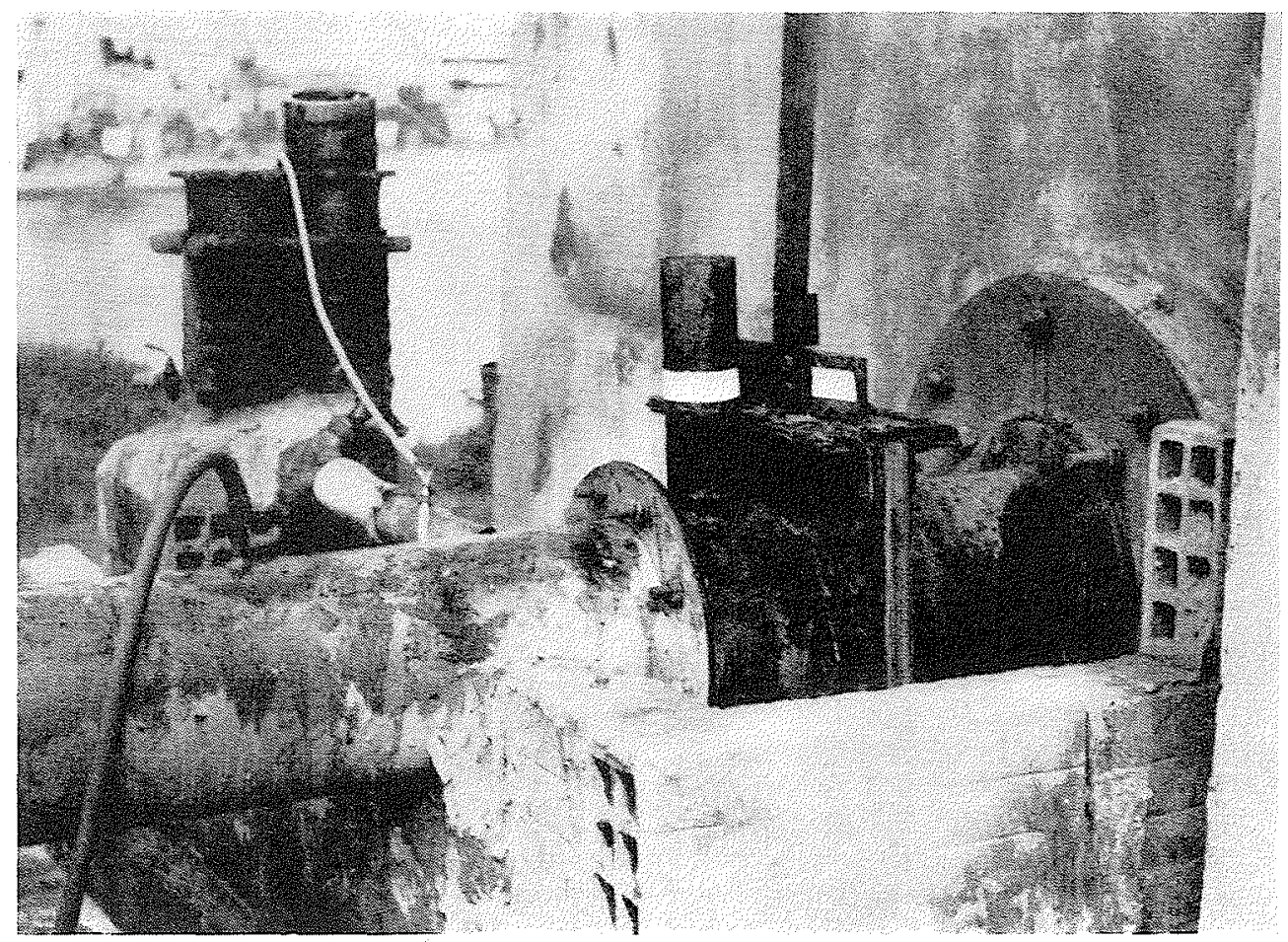

Fig. 25

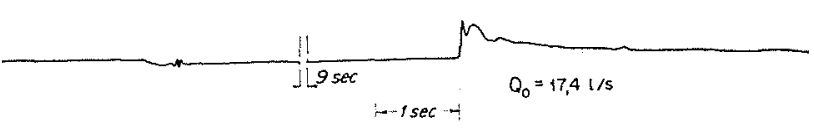

TABLEAU III
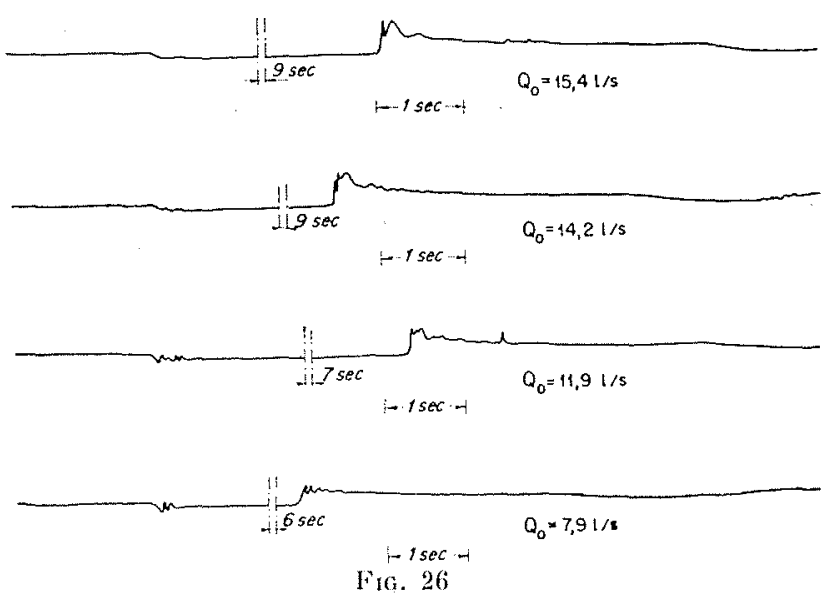

\begin{tabular}{|c|c|c|c|c|}
\hline$Q_{0} l / s$ & $\left|\begin{array}{cc}5_{\mathrm{yr}} & \mathrm{m} \\
\text { expérimental }\end{array}\right|$ & $\begin{array}{l}\text { Sir m } \\
\text { théoligue }\end{array}$ & $\begin{array}{c}\left(t_{1}+t_{2}\right) \mathrm{s} \\
\text { expérimental }\end{array}$ & $\begin{array}{l}\left(t_{1}+t_{2}\right) \mathrm{s} \\
\text { theorique }\end{array}$ \\
\hline 6,1 & 0,363 & 0,397 & 6,1 & 0,8 \\
\hline 7,9 & 0,633 & 0,576 & 7,6 & 8,6 \\
\hline 10,4 & 0,753 & 0,884 & 9,4 & 11,2 \\
\hline 11,9 & 1,073 & 1,094 & 10,1 & 12,8 \\
\hline 11,9 & 0,943 & 1,094 & 10 & 12,8 \\
\hline 11,9 & 0,913 & 1,094 & 10,1 & 12,8 \\
\hline 14,2 & 1,343 & 1,442 & 11,2 & 15,1 \\
\hline 14,2 & 1,233 & 1,442 & 11,2 & 15,1 \\
\hline 15,4 & 1,293 & 1,608 & 11,75 & 16,4 \\
\hline 15,4 & 1,613 & 1,608 & 11,9 & 16,4 \\
\hline 17,4 & 1,453 & 1,973 & 12,76 & 16,4 \\
\hline 17,4 & 1,573 & 1,973 & 12,8 & 16,4 \\
\hline
\end{tabular}


TABLEAU III'

\begin{tabular}{|c|c|c|}
\hline$Q_{0} l / s$ & $\begin{array}{c}\Omega^{\prime} l \\
\text { expérimental }\end{array}$ & $\begin{array}{c}\Omega^{\prime} l \\
\text { théorique }\end{array}$ \\
\hline & & 10,4 \\
10,8 & 10 & 11,6 \\
12,3 & 11,4 & 12,5 \\
13,5 & 11,9 & 13,25 \\
14,6 & 13 & 13,9 \\
15,6 & 13,05 & 14,3 \\
16,3 & 13,5 & 14,8 \\
17,1 & 13,7 & 15,4 \\
18,2 & 14 & \\
\hline
\end{tabular}
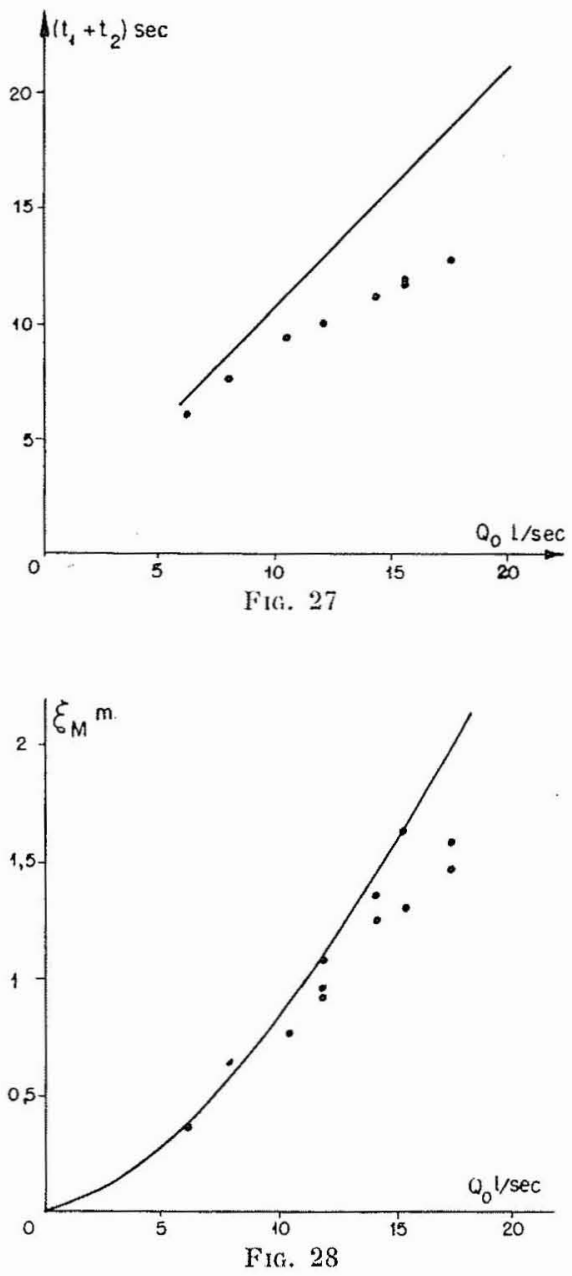

moins bonne pour les durées que pour les surpressions et les débits déversants.

Les écarts s'accentuent d'ailleurs, même pour ces deux derniers éléments, dans le cas des forts débits pour lesquels, il est vrai, une petite aspi-
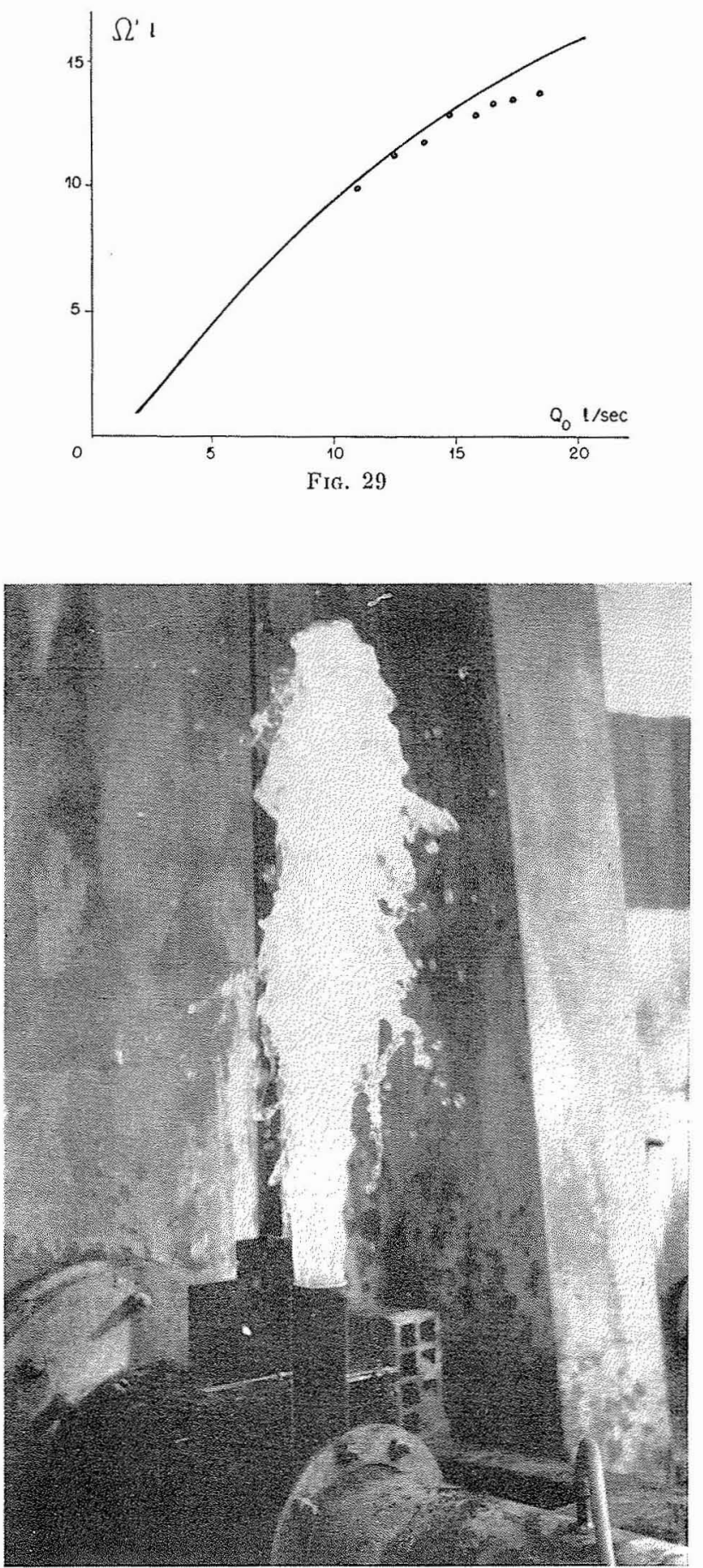

Fig. 30

ration d'air a pu, dans certains cas, se produire par la cheminée, dans le régime permanent initial.

La figure 30 montre le jaillissement de l'eau par la cheminée au début du déversement. 


\section{EXPÉRIENCES DE VISUALISATION}

Nous avons voulu observer directement la formation de la cavité liquide conséculive à la fermeture de la vanne.

Dans ce but, nous avons réalisé un modèle de dimensions plus réduites que le précédent:

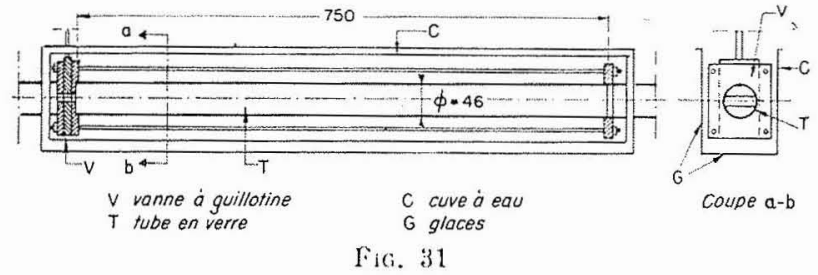

les figures 31 et 32 donnent les dimensions de ce modèle et la photographie de l'extrémité amont; celle-ci est constituée par un tube de verre, d'un diamètre intérieur de $46 \mathrm{~mm}$, placé dans une cuve à eau à parois vitrées verticales dans le but d'éviter la déformation des images due à la courbure du tube.

\section{1. - Visualisation des cavitations.}

Dans le cas de la cavitation, en l'absence de tout dispositif d'aération, des photographies ont été prises au $1 / 1.000^{\circ}$ de seconde : un film a été obtenu avec un einéma M.G.D. à la cadence de 1.700 vues par seconde. Les planches des figures 33 et 34 montrent divers aspects du phénomène enregistrés par la caméra : chaque tour de l'aiguille de l'horloge correspond à une durée de $1 / 10^{\circ}$ de seeonde.
Le film nous a permis de déceler la formation et la disparition de plusieurs cavitations successives avant que le phénomène soit amorti, alors que l'observation directe ne permettait pas de scinder le phénomène d'ensemble, trop rapide, en ses diverses phases.

De façon plus précise, et grâce à l'enregistretrement par l'intermédiaire des photographies de l'horloge, on obtient les valeurs suivantes pour les instants d'apparition et de disparition de cavitations successives:

Apparition de la $1^{\text {re }}$ cavitation $\mathrm{T}=0,0000 \mathrm{~s}$ Disparition de la $1^{\text {re }}$ cavitation $\mathrm{T}=0,1765 \mathrm{~s}$

Apparition de la $2^{\circ}$ cavitation $\mathrm{r}=0,1824 \mathrm{~s}$ Disparition de la $2^{\text {c }}$ cavitation $\mathrm{T}=0,2878 \mathrm{~s}$

Apparition de la $3^{\circ}$ cavitation $\mathrm{T}=0,2976 \mathrm{~s}$ Disparition de la $3^{\circ}$ cavitation $\mathrm{T}=0,3528 \mathrm{~s}$

Apparition de la $4^{\circ}$ cavitation $\mathrm{T}=0,363 \mathrm{~s}$ Disparition de la $4^{\circ}$ cavitation $\mathrm{T}=0,393 \mathrm{~s}$

Apparition de la $5^{\text {e }}$ cavitation $\mathrm{T}=0,405 \mathrm{~s}$ Disparition de la $5^{\circ}$ cavitation $\mathrm{T}=0,431 \mathrm{~s}$

L'amortissement, qui rend déjà impréeises les déterminations relatives à la cinquième cavitation, ne nous a pas permis de pousser plus loin, de façon utile, l’analyse du film.

La figure 33 a montre l'apparition de la première cavitation : il est rappelé que les trois vues successives correspondent à un décalage dans le

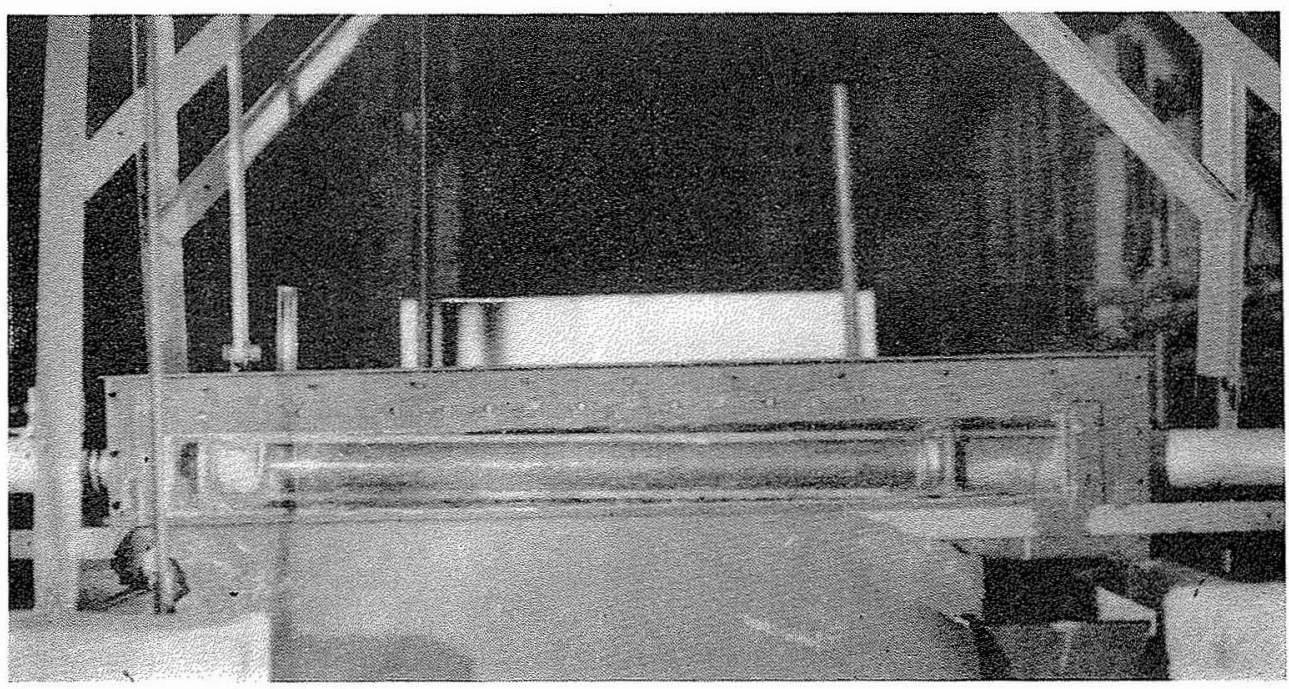

FIG. 32 

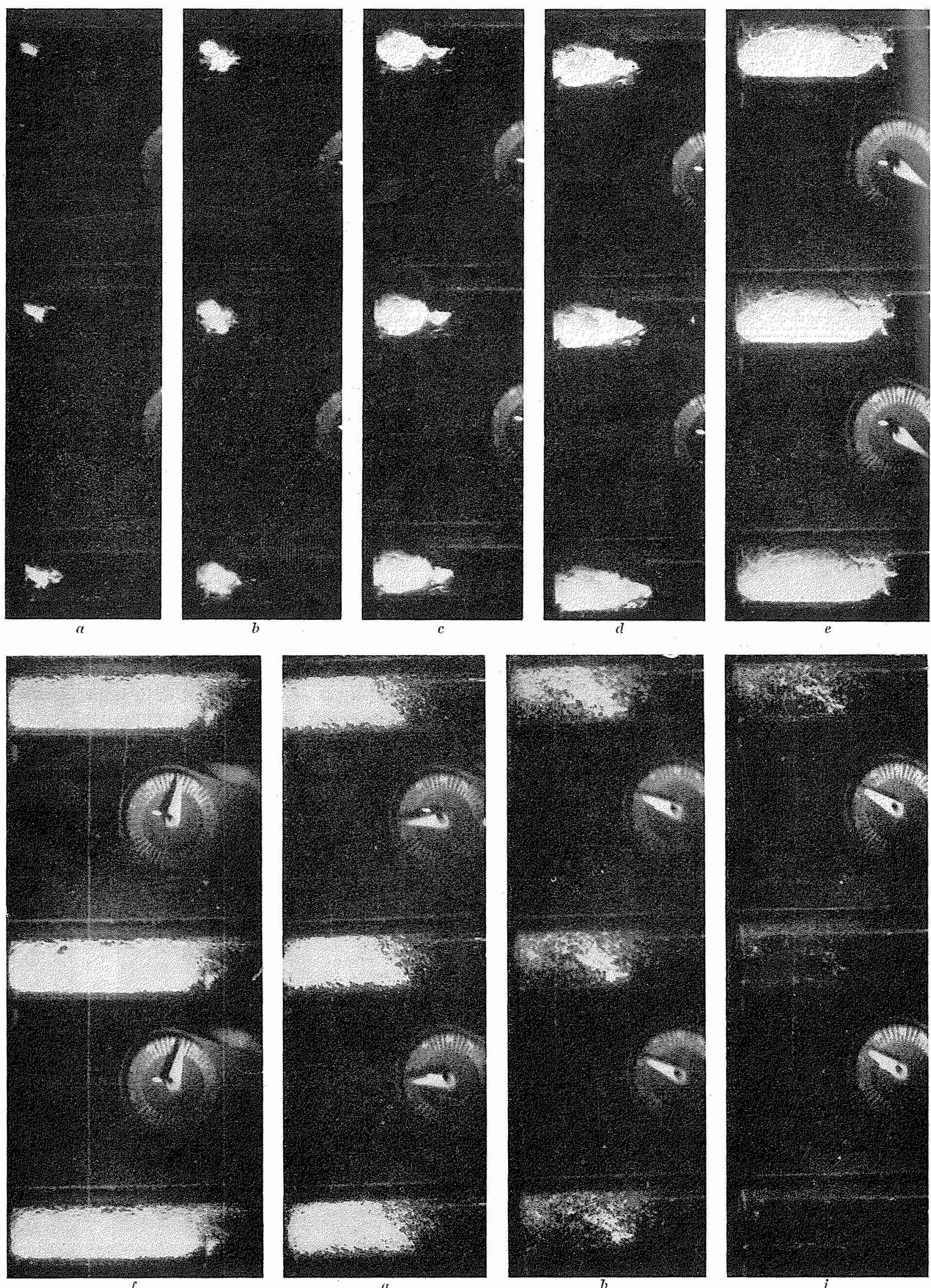


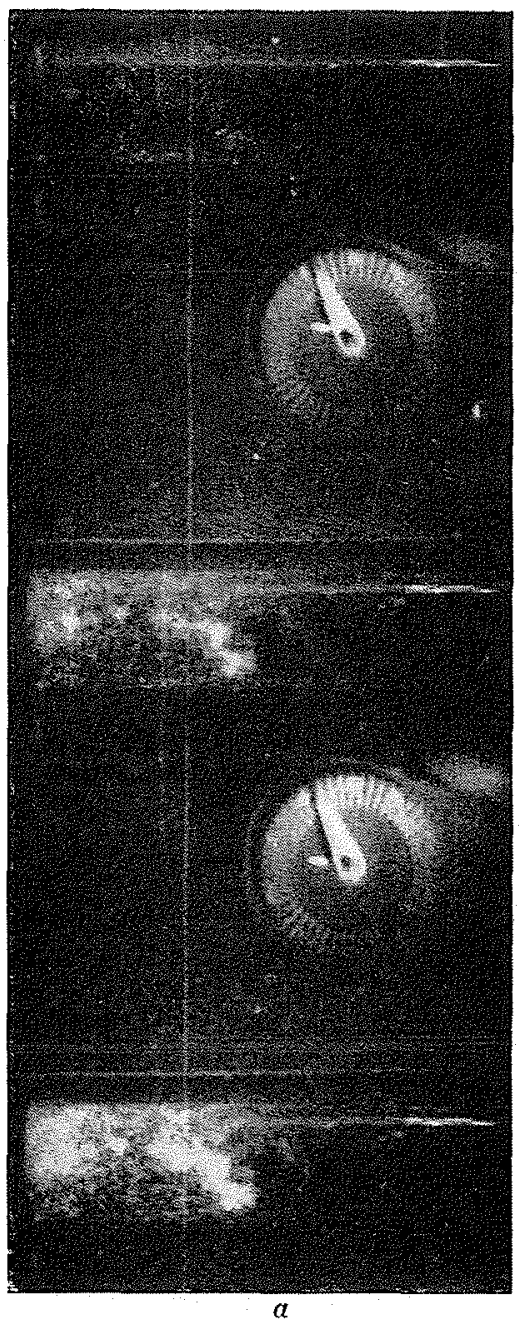

$a$

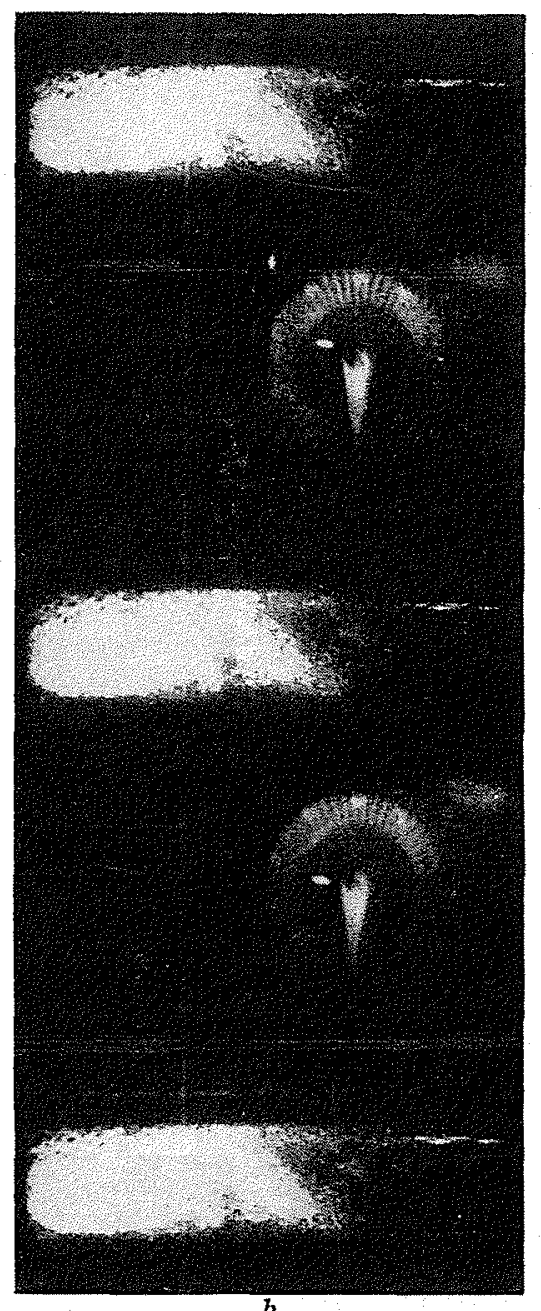

FIG. 34

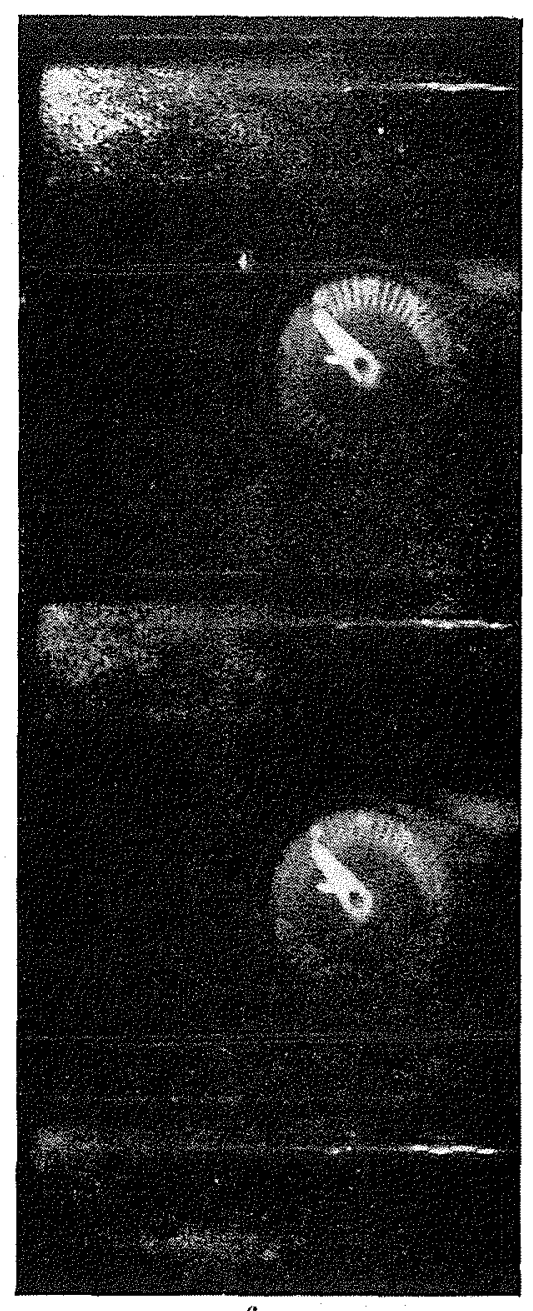

$c$

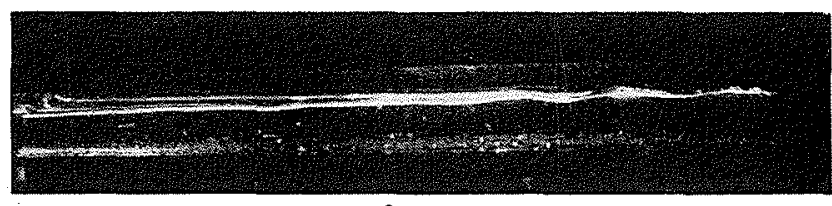

e
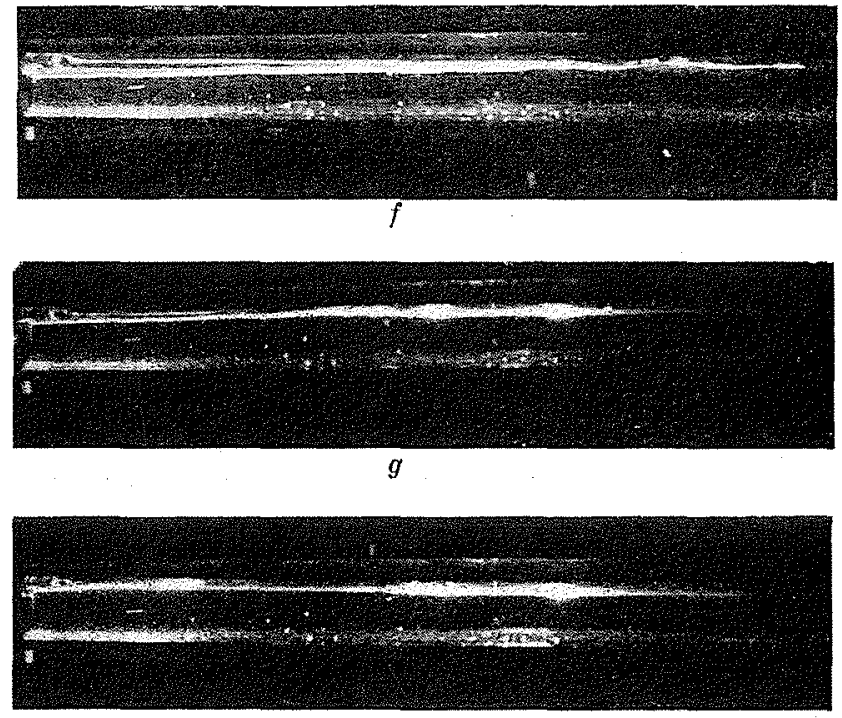

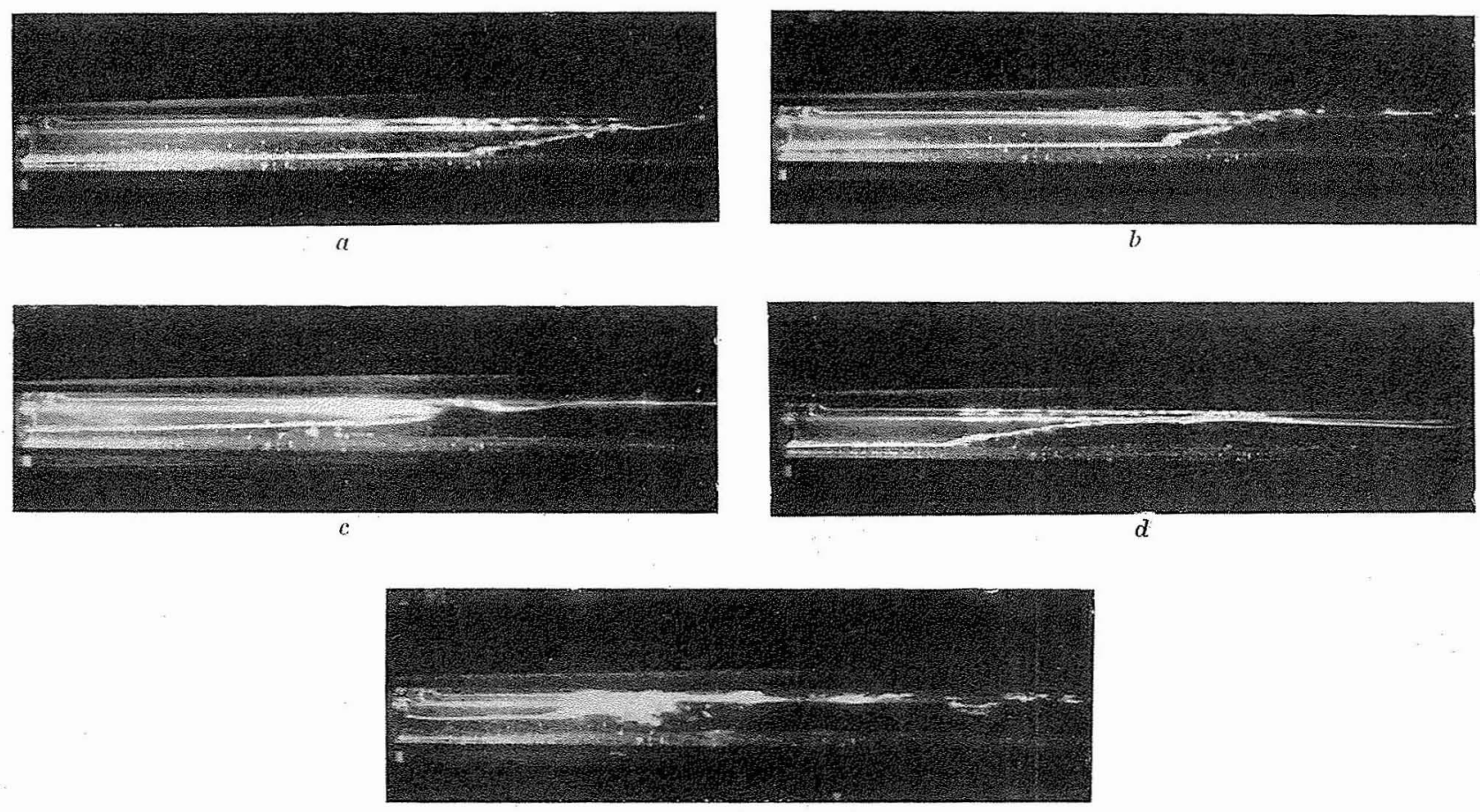

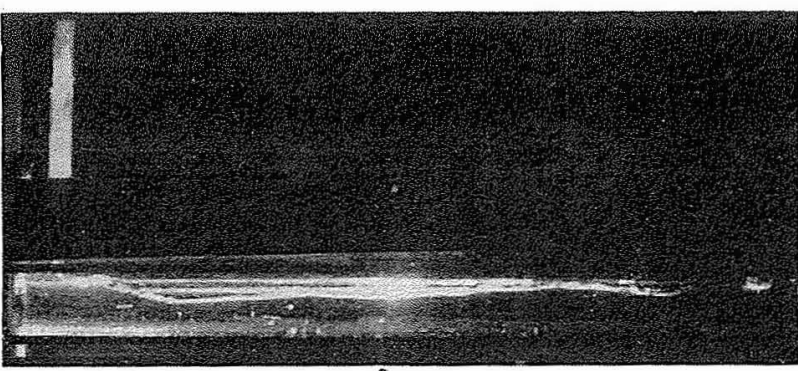

$f$

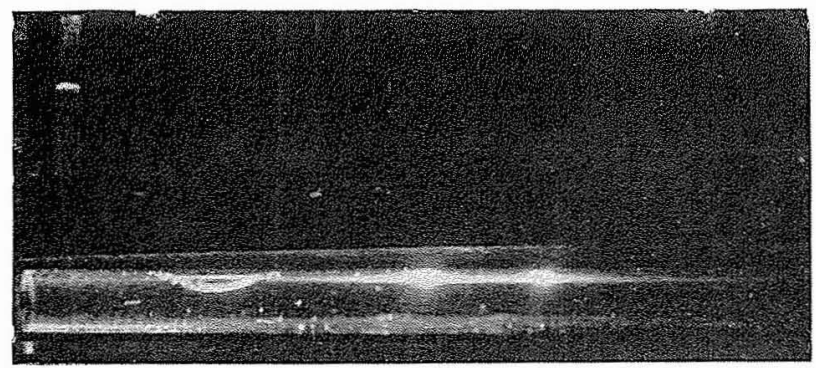

(1)

FIG. 36

temps de $1 / 1.700 \mathrm{~s}$ : on observe la croissance rapide de la cavitation.

Les figures $33 b, 33 c, 33 d, 33 e, 33 f$ permettent de suivre les étapes du développement de la cavité qui atteint sensiblement son maximum sur la figure $33 \mathrm{f}$.

Le phénomène de réduction de la cavité correspond aux photographies des figures successives $33 \mathrm{~g}$, $33 \mathrm{~h}, 33 i$. On remarque, sur la figure $33 i$, que les bulles constituant les cavités élémentaires disparaissent brusquement dans toute la masse.

Sur la figure $34 a$, on voit apparaître brusquement la deuxième cavitation dont le maximum, correspondant sensiblement à la figure $34 \mathrm{~b}$, est moins accentué que celui de la première. La figure $34 c$ enregistre la disparition de cette deuxième cavitation.
Les phénomènes se présentent de facon analogue pour les cavitations suivantes, de plus en plus brèves, réduites et diffuses.

Ajoutons que les mesures ainsi effectuées sur le film sont en bonne concordance avee les prévisions théoriques.

2. - Visualisation dans le cas de l'ateration.

L'extrémité amont du canal est munie d'un tube d'aération à sa partie supérieure.

Le phénomène a été photogyaphié, d'une part, et filmé, d'autre part, au móyen d'une caméra ordinaire, à la cadence normale.

Les huit photographies de la figure 35 montrent la formation, le développement, la rétrac- 
tation et enfin la disparition de la poche d'air, dans le cas d'un débit relativement faible. On observe que la poche d'air se localise à la voute du canal dont la section reste en majeure partie occupée par l'eau.

Au contraire, dans le cas d'une grande vitesse initiale, la colonne liquide est entièrement chassée et une longueur plus ou moins importante du canal est occupée entièrement par l'air, conformément aux hypothèses sur lesquelles se basent les calculs théoriques. On voit, sur la fi- gure 36 , les étapes du phénomène correspondant au retour de l'eau revenant vers l'extrémité amont de la conduite: l'onde à front de plus en plus raide, qui remonte ainsi vers l'amont, visible sur les figures $36 a$ a $36 b$, donne naissance à un jet, jaillissant de la partie supérieure de la cheminée, jet visible sur les figures $36 f$ et $36 \mathrm{~g}$, cependant qu'une petite poche résiduelle d'air, d'importance variable suivant les expériences, se forme momentanément pour se résorber ensuite lentement.

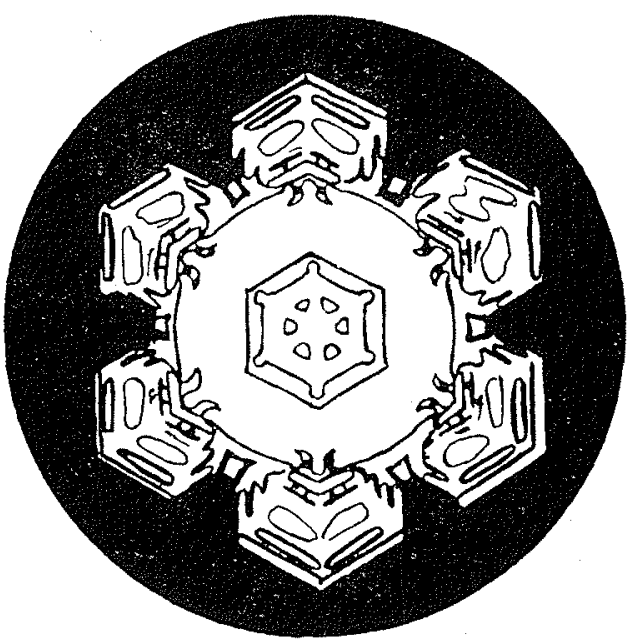

